\title{
PARTIALLY SYMMETRIC SOLUTIONS OF THE GENERALIZED HÉNON EQUATION IN SYMMETRIC DOMAINS
}

\author{
RYUJI KAJIKIYA
}

\begin{abstract}
We study the generalized Hénon equation in a symmetric domain $\Omega$. Let $H$ and $G$ be closed subgroups of the orthogonal group such that $H \varsubsetneqq G$ and $\Omega$ is $G$ invariant. Then we show the existence of a positive solution which is $H$ invariant but $G$ non-invariant under suitable assumptions of $H, G$ and the coefficient function of the equation.
\end{abstract}

\section{Introduction}

We study the existence of partially symmetric positive solutions of the generalized Hénon equation

$$
-\Delta u=f(x) u^{p}, \quad u>0 \quad \text { in } \Omega, \quad u=0 \quad \text { on } \partial \Omega .
$$

Here $\Omega$ is a bounded domain in $\mathbb{R}^{N}$ with piecewise smooth boundary $\partial \Omega, 1<p<$ $\infty$ when $N=2,1<p<(N+2) /(N-2)$ when $N \geq 3, f \in L^{\infty}(\Omega)$ and $f(x)$ may be positive or may change its sign. Let $G$ be a closed subgroup of the orthogonal group $O(N)$. We call $\Omega$ a $G$ invariant domain if $g(\Omega)=\Omega$ for all $g \in G$. We call $f(x)$ a $G$ invariant function if $f(g x)=f(x)$ for all $g \in G$ and $x \in \Omega$. In the same manner, we define a $G$ invariant solution of (1.1). Throughout the paper, we

2010 Mathematics Subject Classification. Primary: 35J20, 35J25.

Key words and phrases. Hénon equation, group invariant solution, least energy solution, positive solution, variational method.

The author was supported in part by the Grant-in-Aid for Scientific Research (C) (No. 24540179), Japan Society for the Promotion of Science. 
assume that the set of $x \in \Omega$ satisfying $f(x)>0$ has positive Lebesgue measure. Otherwise, $f(x) \leq 0$ almost everywhere in $\Omega$ and no positive solution exists by the maximum principle. If $\Omega$ and $f$ are $G$ invariant, (1.1) has a $G$ invariant positive solution, which will be proved in this section. However, we are looking for a positive solution without $G$ invariance. In particular, we investigate the existence of an $H$ invariant $G$ non-invariant solution for closed subgroups $H$ and $G$ such that $H \varsubsetneqq G \subset O(N)$. This solution has a partial symmetry, that is, it is invariant under the $H$ action, but not invariant under the $G$ action.

To get such a solution, we define a least energy solution in the following. First, we introduce the Rayleigh quotient

$$
R(u):=\left(\int_{\Omega}|\nabla u|^{2} d x\right)\left(\int_{\Omega} f(x)|u|^{p+1} d x\right)^{-2 /(p+1)},
$$

with the definition domain

$$
D(R):=\left\{u \in H_{0}^{1}(\Omega): \int_{\Omega} f(x)|u|^{p+1} d x>0\right\} .
$$

Here $H_{0}^{1}(\Omega)$ denotes the Sobolev space. Second, we define the Nehari manifold by

$$
\mathcal{N}:=\left\{u \in H_{0}^{1}(\Omega) \backslash\{0\}: \int_{\Omega}\left(|\nabla u|^{2}-f(x)|u|^{p+1}\right) d x=0\right\} .
$$

Last, we define the least energy $R_{0}$ by

$$
R_{0}:=\inf \{R(u): u \in D(R)\}=\inf \{R(u): u \in \mathcal{N}\} .
$$

The equality of the two infimums above can be proved by using two facts that $R(\lambda u)=R(u)$ for any $\lambda>0$ and $u \in D(R)$; for any $u \in D(R)$ there exists a $\lambda>0$ such that $\lambda u \in \mathcal{N}$. Because of the Sobolev embedding theorem, $R(u)$ has a positive lower bound. Hence $R_{0}$ is well defined and positive. We call $R_{0}$ a least energy and $u$ a least energy solution if $u \in \mathcal{N}$ and $R(u)=R_{0}$. Such a minimizer exists and becomes a solution of (1.1). Note that $R(u)=R(|u|)$. Thus, if $u$ is a least energy solution, so is $|u|$. Then $|u|$ also satisfies (1.1). By the strong maximum principle, $u$ is positive or negative in $\Omega$. Throughout the paper, we choose a positive solution as a least energy solution. A least energy solution is not necessarily unique.

To explain our problem and motivation, we introduce the original Hénon equation

$$
-\Delta u=|x|^{\lambda} u^{p}, \quad u>0 \quad \text { in } B, \quad u=0, \quad \text { on } \partial B,
$$

where $B$ is a unit ball in $\mathbb{R}^{N}$. Smets, Willem and Su [49] have proved that if $\lambda>0$ is large enough, a least energy solution of (1.2) is non-radial. Therefore the equation has at least two positive solutions: a non-radial solution and a radial solution. There are many contributions which have studied the Hénon equation ([4], [7], [10], [12]-[15], [18], [25], [27], [46], [48]). 
The result [49] by Smets, Willem and Su means that if $\lambda$ is large enough, then a least energy solution breaks its radial symmetry. In this case, the weight function $|x|^{\lambda}$ has a thicker density near the boundary $|x|=1$ than the value in the interior. We study the problem above with $B$ replaced by $\Omega$ and $|x|^{\lambda}$ by $f(x)$, where $f(x)$ is a $G$ invariant function and $\Omega$ is a $G$ invariant domain with a closed subgroup $G$ of $O(N)$. Moreover, we look for an $H$ invariant $G$ non-invariant solution. To find such a solution, we define a $G$ invariant least energy solution as below. When $\Omega$ is $G$ invariant, we set

$$
\begin{gathered}
H_{0}^{1}(\Omega, G):=\left\{u \in H_{0}^{1}(\Omega): u \text { is } G \text { invariant }\right\}, \\
D(R, G):=D(R) \cap H_{0}^{1}(\Omega, G), \quad \mathcal{N}(G):=\mathcal{N} \cap H_{0}^{1}(\Omega, G), \\
R_{G}:=\inf \{R(u): u \in D(R, G)\}=\inf \{R(u): u \in \mathcal{N}(G)\} .
\end{gathered}
$$

We call $R_{G}$ a $G$ invariant least energy and $u$ a $G$ invariant least energy solution if $u \in \mathcal{N}(G)$ and $R(u)=R_{G}$. There exists a $G$ invariant least energy solution and it solves (1.1). To avoid confusion, a usual least energy solution is called a global least energy solution.

When $\Omega$ is a hollow thin symmetric domain, we proved in [32] the existence of an $H$ invariant $G$ non-invariant solution. To state this result, we begin with the annulus

$$
A_{N}(a, b):=\left\{x \in \mathbb{R}^{N}: a<|x|<b\right\} .
$$

Coffman [19], Li [34] and Byeon [9] considered the Emden-Fowler equation

$$
-\Delta u=u^{p}, \quad u>0 \quad \text { in } \Omega, \quad u=0 \quad \text { on } \partial \Omega,
$$

with $\Omega=A_{N}(a, b)$ annulus. They proved that the number of non-equivalent positive solutions diverges to infinity as $(b-a) / a \rightarrow 0$. Moreover, a least energy solution is not radially symmetric when $(b-a) / a$ is small enough. Here solutions $u$ and $v$ are said to be equivalent if $u(g x)=v(x)$ with some $g \in O(N)$. For the related results to the annulus, we refer the readers to [2], [16], [17], [21]-[23], [35][40], [45], [50], [51]. Even if $\Omega$ is not an exact annulus but it is like an annular domain without $O(N)$ invariance, the existence of multiple positive solutions was proved by Byeon and Tanaka [11]. For the related results, we refer the readers to [1], [5], [24].

More general equations than (1.4) were studied by papers [6], [33], [43]. Indeed, the existence of a sequence of sign-changing solutions for the critical polyharmonic equation was proved by Bartch, Schneider and Weth [6]. For the $p$-Laplace equation in $\mathbb{R}^{N}$, Kristály and Marzantowicz [33] proved the existence of multiple sequences of sign-changing solutions. Musso, Pacard and Wei [43] proved the existence of a sequence of nonradial sign-changing solutions for a semilinear elliptic equation. 
We shall state our earlier result. Let $\Omega$ be a hollow regular polygon, which is defined as below. Let $P$ be an interior of a regular $n$ polygon with center origin. We define

$$
(1+\varepsilon) P:=\{(1+\varepsilon) x: x \in P\}, \quad \text { with } \varepsilon>0,
$$

which is a regular polygon larger than $P$. We remove $\bar{P}$ from $(1+\varepsilon) P$ and define $\Omega:=(1+\varepsilon) P \backslash \bar{P}$. Then $\Omega$ is a hollow polygon. We define

$$
r(\theta):=\left(\begin{array}{rr}
\cos \theta & -\sin \theta \\
\sin \theta & \cos \theta
\end{array}\right)
$$

$$
G_{n}:=\{r(2 j \pi / n): j=0,1, \ldots, n-1\} .
$$

Then $\Omega$ is $G_{n}$ invariant. Let $1=n_{1}<\ldots<n_{d}=n$ be all divisors of $n$ and let $u_{i}$ be a $G_{n_{i}}$ invariant least energy solution of (1.4). Then we proved in [32] that for $\varepsilon>0$ small enough, $u_{i}$ is not equivalent to $u_{j}$ if $i \neq j$. This is caused by the assumption that $\Omega$ is a hollow thin domain.

Replace (1.4) by (1.1). In this case, we do not need $\Omega$ to have a hole. Instead of a hole, we assume that the ratio of the density of $f(x)$ near $\partial \Omega$ to that of the interior of $\Omega$ is large enough. Then we shall prove the same result as above in Example 3.3.

For a closed subgroup $G$ of $O(N)$ and $x \in \mathbb{R}^{N}$, we define the orbit of $G$ through $x$ by

$$
G(x):=\{g x: g \in G\} .
$$

Let $H$ and $G$ be closed subgroups of $O(N)$ satisfying $H \varsubsetneqq G$. The purpose of this paper is to prove that no $H$ invariant least energy solution is $G$ invariant if $H(x) \varsubsetneqq G(x)$ for all $x \in \Gamma$. Here $H(x)$ and $G(x)$ are the orbits of $H$ and $G$, respectively, and $\Gamma$ is the set of points farthest in $\bar{\Omega}$ from the fixed point set of $G$. By choosing several pairs of $H$ and $G$, we obtain multiple positive solutions of (1.1).

This paper is organized into six sections. In Section 2, we state the main results. In Section 3, we give several examples of symmetric domains $\Omega$ and groups $H, G$ and explain how to apply our theorem to the problem. In Section 4, we give a few lemmas to prove the main results. In Section 5 , we give an a priori $L^{\infty}(\Omega)$ estimate of a $G$ invariant least energy solution and investigate the $L^{2}(\Omega)$ norm. In Section 6, we prove the main theorems.

\section{Main results}

In this section, we state the main results. We define the fixed point set of $G$ by $F=\operatorname{Fix}(G):=\left\{x \in \mathbb{R}^{N}: g x=x\right.$ for all $\left.g \in G\right\}$. Then $F$ is a linear subspace of $\mathbb{R}^{N}$. Let $F^{\perp}$ denote the orthogonal complement of $F$ in $\mathbb{R}^{N}$. Hereafter, the 
notation $x=x^{\prime}+x^{\prime \prime}$ stands for the orthogonal decomposition of $x \in \mathbb{R}^{N}$ with $x^{\prime} \in F$ and $x^{\prime \prime} \in F^{\perp}$. We define a notation

$$
\Omega\left(s<\left|x^{\prime \prime}\right|<t\right):=\left\{x^{\prime}+x^{\prime \prime} \in \Omega: s<\left|x^{\prime \prime}\right|<t\right\} .
$$

Similarly, we define $\Omega\left(\left|x^{\prime \prime}\right|<t\right)$. We put

$$
\begin{array}{r}
\operatorname{dist}(x, F):=\inf \{|x-y|: y \in F\}, \\
A:=\max _{x \in \bar{\Omega}} \operatorname{dist}(x, F)=\max _{x \in \partial \Omega} \operatorname{dist}(x, F) .
\end{array}
$$

We denote the set of points farthest in $\bar{\Omega}$ from $F$ by $\Gamma$, i.e.

$$
\Gamma:=\{x \in \partial \Omega: \operatorname{dist}(x, F)=A\},
$$

where $A$ is given by (2.1).

If $f \geq 0, \not \equiv 0$ in $\Omega$, we define, for $N \geq 3$ and $A_{0} \in(0, A)$,

$$
\mu\left(f, A_{0}\right):=\|f\|_{L^{\infty}\left(\Omega\left(\left|x^{\prime \prime}\right|<A_{0}\right)\right)}^{N+2-(N-2) p}\|f\|_{L^{\infty}(\Omega)}^{(N-2)(p-1)}\left(\int_{\Omega} f(x) \operatorname{dist}(x, \partial \Omega)^{p+1} d x\right)^{-4},
$$

and for $N=2$ with $\theta \in(0,1)$,

$$
\mu\left(f, A_{0}, \theta\right):=\|f\|_{L^{\infty}\left(\Omega\left(\left|x^{\prime \prime}\right|<A_{0}\right)\right)}\|f\|_{L^{\infty}(\Omega)}^{(1-\theta) / \theta}\left(\int_{\Omega} f(x) \operatorname{dist}(x, \partial \Omega)^{p+1} d x\right)^{-1 / \theta} .
$$

Here $\|\cdot\|_{L^{q}(\Omega)}$ denotes the $L^{q}(\Omega)$ norm and $\operatorname{dist}(x, \partial \Omega)$ is the distance function from $x$ to $\partial \Omega$.

Let $G$ and $H$ be closed subgroups of $O(N)$ such that $\Omega$ is $G$ invariant and $H \varsubsetneqq G$. We introduce an assumption

$$
H(x) \varsubsetneqq G(x) \text { for all } x \in \Gamma .
$$

Here $H(x)$ and $G(x)$ denote the orbits defined by (1.6) and $\Gamma$ is defined by (2.2). Define $f_{+}(x):=\max (f(x), 0)$. We consider the following two conditions separately.

(A) $f(x) \leq 0$ in $\Omega\left(\left|x^{\prime \prime}\right|<A_{0}\right)$ and $f_{+}(x) \not \equiv 0$ in $\Omega\left(A_{0}<\left|x^{\prime \prime}\right|<A\right)$.

(B) $f(x) \geq 0, \not \equiv 0$ in $\Omega$ and $\mu\left(f, A_{0}\right)$ is small enough when $N \geq 3$ and $\mu\left(f, A_{0}, \theta\right)$ with a certain $\theta \in(0,1)$ is small enough when $N=2$.

Recall that $R_{G}$ is defined by (1.3). We state the first main result.

TheOREM 2.1. Let $G$ and $H$ be closed subgroups of $O(N)$ such that $\Omega$ is $G$ invariant, $H \varsubsetneqq G$ and they satisfy (2.3). Then there exists an $\varepsilon \in(0, A)$ independent of $f(x)$ such that if either $(\mathrm{A})$ or $(\mathrm{B})$ holds at some $A_{0} \in(A-\varepsilon, A)$, then $R_{H}<R_{G}$. Therefore if $u$ is an $H$ invariant least energy solution, it is not $G$ invariant. Thus (1.1) has at least two positive solutions: one is an $H$ invariant $G$ non-invariant positive solution and another is a $G$ invariant positive solution. 
Denote the set of $G$ invariant continuous functions on $\bar{\Omega}$ by $C(\bar{\Omega}, G)$. Instead of assumption (B), we consider the next one.

(B)' $f(x):=f_{0}(x)^{\lambda}$ with $\lambda>0$, where $f_{0} \in C(\bar{\Omega}, G)$ satisfies

$$
0 \leq f_{0}(x)<\max _{y \in \Gamma} f_{0}(y) \quad \text { for } x \in \bar{\Omega} \backslash \Gamma .
$$

Then we have the next corollary.

Corollary 2.2. Let $G, H$ and $\Omega$ be as in Theorem 2.1. Let $f \in C(\bar{\Omega}, G)$ satisfy either assumption (A) with $A_{0}$ sufficiently close to $A$ or assumption (B)' with $\lambda>0$ large enough. Then no $H$ invariant least energy solution of (1.1) can be $G$ invariant.

Now, we consider the case where $\operatorname{Fix}(G)=\{0\}$. This condition is satisfied if, for example, $G=\{I,-I\}$ with the unit matrix $I, G=G_{n}$ defined by (1.5) or $G=O(N)$, etc. Then $A$ defined by (2.1) is the radius of the circumscribed sphere of $\Omega$ and $\Gamma$ is the intersection of this sphere and $\partial \Omega$. Let $f(r)$ with $r=|x|$ be radially symmetric. Then assumption (A) is rewritten as

$$
f(r) \leq 0 \quad \text { in }\left(0, A_{0}\right), \quad f_{+}(r) \not \equiv 0 \quad \text { in }\left(A_{0}, A\right) .
$$

Condition (2.4) is reduced to

$$
0 \leq f_{0}(r)<f_{0}(A) \text { for } 0 \leq r<A,
$$

where $f(r)=f_{0}(r)^{\lambda}$ and $f_{0} \in C[0, A]$. Then Corollary 2.2 leads to the next one.

Corollary 2.3. Let $G, H$ and $\Omega$ be as in Theorem 2.1. Moreover, assume that $\operatorname{Fix}(G)=\{0\}$. Let $f(r)$ with $r=|x|$ be a radial continuous function satisfying either (2.5) with $A_{0} \in(0, A)$ sufficiently close to $A$ or (2.6) with $f(r):=f_{0}(r)^{\lambda}$ and $\lambda$ large enough. Then no $H$ invariant least energy solution of (1.1) is $G$ invariant.

There are various functions $f(|x|)$ satisfying (2.6), e.g. $f(|x|)=|x|^{\lambda}$ (the original Hénon equation), $f(|x|)=e^{\lambda|x|},(|x| /(1+|x|))^{\lambda}$ and $(\sin (\pi|x| / 2 A))^{\lambda}$, etc. The simplest example satisfying $(2.5)$ is $f(|x|)=|x|-A_{0}$.

REMARK 2.4. We mention that (2.3) is related to the necessary condition for the existence of an $H$ invariant $G$ non-invariant solution. Suppose that

$$
H(x)=G(x) \text { for all } x \in \Omega .
$$

Under this condition, any $H$ invariant function coincides with a $G$ invariant function. Therefore the existence of an $H$ invariant $G$ non-invariant solution needs the condition,

$$
H(x) \varsubsetneqq G(x) \text { at some } x \in \Omega .
$$

This is a necessary condition and (2.3) is stronger than this condition. 
Since $G$ is an isometric transformation group on $\mathbb{R}^{N}$, it becomes that on the unit sphere $S^{N-1}$,

$$
S^{N-1}:=\left\{x \in \mathbb{R}^{N}:|x|=1\right\} .
$$

If $G(x)=S^{N-1}$ for $x \in S^{N-1}, G$ is said to be transitive on $S^{N-1}$. If $G$ is transitive, then a $G$ invariant solution becomes radially symmetric. If $G$ is not transitive, then $G(x) \varsubsetneqq S^{N-1}=O(N)(x)$ for $x \in S^{N-1}$, which implies that $G(x) \varsubsetneqq O(N)(x)$ for all $x \neq 0$. This condition means that the pair $(G, O(N))$ satisfies (2.3). Then Corollary 2.3 implies the next result.

Corollary 2.5. Let $\Omega$ be an annulus or a ball, i.e.

$$
\Omega:=\left\{x \in \mathbb{R}^{N}: a<|x|<A\right\} \quad \text { or } \quad \Omega:=\left\{x \in \mathbb{R}^{N}:|x|<A\right\} .
$$

Let $f(|x|)$ be a radial continuous function satisfying either (2.5) with $A_{0}$ close to $A$ or (2.6) with $\lambda>0$ large enough. Let $G$ be a closed subgroup of $O(N)$. Then the following two assertions are equivalent.

(a) $G$ is not transitive on $S^{N-1}$.

(b) No G invariant least energy solution can be radially symmetric.

REMARK 2.6. In our paper [28], we studied sign-changing solutions of (1.1) in a ball and an annulus, where we took $f(x) \equiv 1$ and replaced $u^{p}$ by $|u|^{p-1} u$. Then we proved that (a) in Corollary 2.5 is equivalent to the existence of a sequence $u_{k}$ of sign-changing solutions which are $G$ invariant but non-radial and the $H_{0}^{1}(\Omega)$ norm of $u_{k}$ diverges to infinity as $k \rightarrow \infty$.

All transitive Lie groups have been classified by Montgomery and Samelson [41] and Borel [8] as below (see also [26, p. 186, Theorem 2.6] and [44, p. 267, Theorem 3]).

Theorem 2.7 (Montgomery and Samelson [41], Borel [8]). Let $N \geq 2$ and $G$ be a connected closed subgroup of $S O(N)$. Here $S O(N)$ is the special orthogonal group (rotation group). Then $G$ is transitive on $S^{N-1}$ if and only if $G$ is locally isomorphic to one of the following groups: $S O(N)$; $S U(m), U(m)$ if $N=2 m$; $S p(m), S p(m) S p(1), S p(m) U(1)$ if $N=4 m$; $\operatorname{Spin}(9)$ if $N=16$; $\operatorname{Spin}(7)$ if $N=8 ; G_{2}$ if $N=7$.

When $G$ is not necessarily connected, we denote by $G_{0}$ the connected component of $G$ which has the unit matrix. Then $G_{0}$ becomes a closed subgroup of $G$. Moreover, $G$ is transitive if and only if so is $G_{0}$. Therefore a closed subgroup $G$ of $O(N)$ is transitive if and only if $G_{0}$ is $O(N)$ locally isomorphic to one of the groups listed in the theorem above. Since a finite subgroup of $O(N)$ is not transitive, we have the following result. 
Corollary 2.8. Let $\Omega$ and $f$ be as in Corollary 2.5 and let $G$ be a finite subgroup of $O(N)$. Then no $G$ invariant least energy solution can be radially symmetric.

ExAmPLE 2.9. Let $\Omega$ and $f$ be as in the corollary above. Then an even least energy solution is not radially symmetric. Indeed, we choose $G=\{I,-I\}$ with the unit matrix $I$. Then a $G$ invariant function is even. Therefore Corollary 2.8 shows the conclusion.

\section{Examples}

In this section, we give several examples of $G, H$ and $\Omega$ and demonstrate how to apply our theorem to the problem. Then we obtain multiple positive solutions.

EXAMPLE 3.1. Let $\Omega$ be a bounded and point symmetric domain, i.e., $x \in \Omega$ implies $-x \in \Omega$. Put $A:=\max _{x \in \bar{\Omega}}|x|$. Let $f(x)$ be a continuous even function on $\bar{\Omega}$ satisfying either $(\mathrm{A})$ or $(\mathrm{B})$ below.

(A) $f(x) \leq 0$ in $\Omega\left(0<|x|<A_{0}\right)$ and $f_{+}(x) \not \equiv 0$ in $\Omega\left(A_{0}<|x|<A\right)$.

(B) $f(x)=f_{0}(x)^{\lambda}$, where $f_{0}(x)$ is a continuous even function satisfying $0 \leq$ $f_{0}(x)<\max _{|y|=A} f_{0}(y)$ for $0 \leq|x|<A$.

If (A) holds with $A_{0}$ sufficiently close to $A$ or if (B) holds with $\lambda>0$ large enough, then a global least energy solution is not even. Therefore (1.1) has both an even positive solution and a non-even positive solution. We shall show this claim. Put $H:=\{I\}$ and $G:=\{I,-I\}$ with the unit matrix $I$. Then $\operatorname{Fix}(G)=\{0\}$ and $(2.3)$ holds. In this case, an $H$ invariant least energy solution coincides with a global least energy solution. Corollary 2.2 shows that a global least energy solution is not even.

EXAmple 3.2. Let $N=2$ and $\Omega$ be a square defined by

$$
\Omega:=\left\{\left(x_{1}, x_{2}\right) \in \mathbb{R}^{2}:\left|x_{1}\right|<1,\left|x_{2}\right|<1\right\} .
$$

Let $f(r)$ with $r=|x|$ be a radial continuous function defined for $r \in[0, \sqrt{2}]$ and hence it is defined on the whole $\Omega$. Assume that $f(r)$ satisfies either (2.5) with $A_{0}$ slightly less than $\sqrt{2}$ or $(2.6)$ with $\lambda>0$ large enough. Let $H:=\{I,-I\}$ with the unit matrix $I$ and define

$$
G:=\left\{\left(\begin{array}{ll}
1 & 0 \\
0 & 1
\end{array}\right),\left(\begin{array}{rr}
-1 & 0 \\
0 & 1
\end{array}\right),\left(\begin{array}{rr}
1 & 0 \\
0 & -1
\end{array}\right),\left(\begin{array}{rr}
-1 & 0 \\
0 & -1
\end{array}\right)\right\} .
$$

Then $G$ is a reflection group with respect to the $x$-axis and the $y$-axis. $H$ is a point reflection with respect to the origin. Clearly, $H$ is a subgroup of $G$. It holds that $\operatorname{Fix}(G)=\{0\}$ and $\Gamma$ consists of four vertices of $\Omega$, i.e.

$$
\Gamma=\{(1,1),(1,-1),(-1,1),(-1,-1)\},
$$


The constant $A$ defined by (2.1) is equal to $\sqrt{2}$, the radius of the circumscribed circle of $\Omega$. From the definition of the orbit, it follows that

$$
H(x)=\{x,-x\}, \quad G(x)=\Gamma \quad \text { for } x \in \Gamma,
$$

which means that $H(x) \varsubsetneqq G(x)$ for all $x \in \Gamma$. Thus $(H, G, \Omega)$ satisfies (2.3). By Corollary 2.2, a point symmetric (i.e. even) least energy solution has no reflective symmetry with respect to either the $x$-axis or the $y$-axis. Observe that a combination of a point symmetry and the $x$-axis reflective symmetry yields the $y$-axis reflective symmetry. This claim is still valid by exchanging $x$ with $y$. Therefore our theorem says that a point symmetric least energy solution has neither the $x$-axis reflective symmetry nor the $y$-axis reflective symmetry.

In the following, we consider various groups $G$. Note that $\Gamma$ defined by $(2.2)$ depends only on $\Omega$ and $G$. Since $\Omega$ is fixed, we denote $\Gamma$ by $\Gamma(G)$. Let $G_{4}$ and $H$ be as in (1.5) and as above, respectively. Then $H$ is a subgroup of $G_{4}$. It is easy to compute that $\operatorname{Fix}\left(G_{4}\right)=\{0\}$ and $\Gamma\left(G_{4}\right)$ is equal to $\Gamma$ in (3.1). Hence $\left(H, G_{4}, \Omega\right)$ satisfies (2.3). Accordingly, a point symmetric least energy solution is not invariant under the rotation by angle $\pi / 4$.

Define

$$
K:=\left\{\left(\begin{array}{ll}
1 & 0 \\
0 & 1
\end{array}\right),\left(\begin{array}{ll}
0 & 1 \\
1 & 0
\end{array}\right),\left(\begin{array}{rr}
0 & -1 \\
-1 & 0
\end{array}\right),\left(\begin{array}{rr}
-1 & 0 \\
0 & -1
\end{array}\right)\right\} .
$$

Then $K$ is the reflection with respect to the lines $y=x$ and $y=-x . H$ is a subgroup of $K$ and $\Gamma(K)$ is equal to $\Gamma$ in (3.1). In this case, $(H, K, \Omega)$ does not satisfy (2.3). Indeed, we have

$$
H(x)=K(x)=\{x,-x\} \quad \text { for } x \in \Gamma .
$$

The square $\Omega$ has four lines of symmetry: $x$-axis, $y$-axis, $y=x$ and $y=-x$. Our theorem ensures that a point symmetric least energy solution does not have any symmetries of the $x$-axis reflection, the $y$-axis reflection and the rotation by $\pi / 4$. However our theorem says nothing about whether a point symmetric least energy solution has no reflective symmetry with respect to the line $y=x$ or $y=-x$.

We consider a global least energy solution. Let $H=\{I\}$ and $G=\{I,-I\}$. Then an $H$ invariant least energy solution is a global least energy solution. For this $G, \Gamma(G)$ coincides with $\Gamma$ in (3.1). Since $H(x) \varsubsetneqq G(x)$ for $x \in \Gamma$, a global least energy solution is not even. We shall show that a global least energy solution is not symmetric with respect to the $x$-axis reflection. Let $H:=\{I\}$ and define

$$
G:=\left\{\left(\begin{array}{rr}
1 & 0 \\
0 & 1
\end{array}\right),\left(\begin{array}{rr}
1 & 0 \\
0 & -1
\end{array}\right)\right\} .
$$


Then $G$ is the reflection group of the $x$-axis. Then $\operatorname{Fix}(G)$ is the $x$-axis and hence

$$
\Gamma(G)=\left\{\left(x_{1}, x_{2}\right):-1 \leq x_{1} \leq 1, x_{2}= \pm 1\right\} .
$$

We compute

$$
H(x)=\{x\}, \quad G(x)=\left\{\left(x_{1}, x_{2}\right),\left(x_{1},-x_{2}\right)\right\} \quad \text { for } x=\left(x_{1}, x_{2}\right) \in \Gamma(G) .
$$

Therefore $(H, G)$ satisfies $(2.3)$. Since $\operatorname{Fix}(G)$ is the $x$-axis, we compute

$$
\Omega\left(\left|x^{\prime \prime}\right|<A_{0}\right)=\left\{\left(x_{1}, x_{2}\right) \in \Omega:\left|x_{2}\right|<A_{0}\right\} .
$$

At the beginning of the example, we defined a radial continuous function $f(r)$. For $G$ above, $f(r)$ still satisfies (A) or (B)', which can be proved by using the expression of $\Omega\left(\left|x^{\prime \prime}\right|<A_{0}\right)$ above. Hence a global least energy solution is not symmetric with respect to the $x$-axis reflection. In the same way, we see that a global least energy solution does not have any symmetries of the $x$-axis reflection, the $y$-axis reflection and the rotation by $\pi / 4$.

We have already stated in Introduction that when $\Omega$ is a hollow thin polygon, (1.4) has an $H$ invariant $G$ non-invariant solution. We consider the usual polygon without a hole in the next example.

EXAMPLE 3.3. Let $\Omega$ be a regular $n$ polygon with center origin. Let $1=$ $n_{1}<\ldots<n_{d}=n$ be all the divisors of $n$ and $u_{i}$ be a $G_{n_{i}}$ invariant least energy solution. Here $G_{n}$ is defined by (1.5). Let $A$ be the distance between the center and a vertex of $\Omega$, i.e., the radius of the circumscribed circle of $\Omega$. Let $f(x)$ be a $G_{n}$ invariant function satisfying either (A) in Example 3.1 with $A_{0}$ sufficiently close to $A$ or (B) with $\lambda>0$ large enough. Then $u_{i}$ is not equivalent to $u_{j}$ for $i \neq j$. For example, consider the regular hexagon $\Omega$. Then it is $G_{6}$ invariant. All subgroups of $G_{6}$ are $G_{1}, G_{2}, G_{3}$ and $G_{6}$. Let $u_{i}$ be a $G_{i}$ invariant least energy solution. Then $u_{1}, u_{2}, u_{3}$ and $u_{6}$ are not equivalent to each other.

The above example and the next one will be proved in the last section.

EXAMPLE 3.4. Let $\Omega$ be a cylinder in $\mathbb{R}^{3}$, which is defined by

$$
\Omega:=\left\{\left(x_{1}, x_{2}, x_{3}\right): x_{1}^{2}+x_{2}^{2}<\alpha^{2},\left|x_{3}\right|<\beta\right\},
$$

with $\alpha, \beta>0$. Let $f(x)$ be a continuous function which is rotationally symmetric around the $x_{3}$ axis and satisfies either (A) or (B) below.

(A) $f(x) \leq 0$ in $\Omega\left(\left|\left(x_{1}, x_{2}\right)\right|<a\right)$ and $f_{+}(x) \not \equiv 0$ in $\Omega\left(a<\left|\left(x_{1}, x_{2}\right)\right|<\alpha\right)$.

(B) $f\left(x_{1}, x_{2}, x_{3}\right)=f_{0}\left(\left|\left(x_{1}, x_{2}\right)\right|\right)^{\lambda}$, where $f_{0}\left(\left|\left(x_{1}, x_{2}\right)\right|\right)$ is a radial function satisfying $0 \leq f_{0}(t)<f_{0}(\alpha)$ for $0 \leq t=\left|\left(x_{1}, x_{2}\right)\right|<\alpha$.

Then the number of non-equivalent positive solutions diverges to infinity as $a \rightarrow$ $\alpha$ in Case (A) or $\lambda \rightarrow \infty$ in Case (B). 
EXAMPLE 3.5. Let $\Omega$ be an interior of a regular tetrahedron centered at the coordinate origin $O$ in $\mathbb{R}^{3}$. Let $A$ be the radius of the circumscribed sphere of $\Omega$. Let $f(r)$ with $r=|x|$ be a radial continuous function defined on $r \in[0, A]$. Hence it is well defined on the whole $\Omega$. Suppose that $f(r)$ satisfies either $(2.5)$ with $A_{0}$ sufficiently close to $A$ or (2.6) with $\lambda>0$ large enough. Then (1.1) has at least 3 non-equivalent positive solutions. We shall show this claim. We denote the symmetry group of $\Omega$ by $O(\Omega)$ and the rotational symmetry group by $S O(\Omega)$, which are defined by

$$
O(\Omega):=\{g \in O(3): g(\Omega)=\Omega)\}, \quad S O(\Omega):=\{g \in S O(3): g(\Omega)=\Omega)\}
$$

Then it is known that $S O(\Omega)$ is isomorphic to the alternating group $A_{4}$, and $O(\Omega)$ to the symmetric group $S_{4}$ (for the proof, see [20, p. 49] or [3, pp. 37-38]). Denote the unit element in $S_{4}$ by $e$. Then all subgroups of $S_{4}$ which are not conjugate to each other are listed below.

$$
\begin{aligned}
G_{1} & :=\{e\} \\
G_{2} & :=\{e,(12)\} \\
G_{3} & :=\{e,(12)(34)\} \\
G_{4} & :=\{e,(123),(132)\} \\
G_{5} & :=\{e,(1324),(12)(34),(1423)\} \\
G_{6} & :=\{e,(12),(34),(12)(34)\} \\
G_{7} & :=\{e,(12)(34),(13)(24),(14)(23)\} \\
G_{8} & :=S_{3}=\{e,(12),(13),(23),(123),(132)\}, \\
G_{9} & :=\{e,(12),(34),(12)(34),(13)(24),(14)(23),(1324),(1423)\}, \\
G_{10} & :=A_{4}, \\
G_{11} & :=O(\Omega)=S_{4} .
\end{aligned}
$$

Let $O$ be the center of the tetrahedron $\Omega$ and $V_{i}$ with $1 \leq i \leq 4$ be vertices of $\Omega$. Let $V_{i j}$ be the middle point of the edge connecting $V_{i}$ with $V_{j}$. Let $L_{12}$ denote the reflection matrix with respect to the plane through three points $V_{12}, V_{3}$ and $V_{4}$. This reflection leaves the tetrahedron $\Omega$ invariant and interchanges $V_{1}$ with $V_{2}$, but leaves $V_{3}$ and $V_{4}$ invariant. Thus $L_{12}$ corresponds to the transposition (12). For simplicity, we write $L_{12}=(12)$. Similarly, we can define $L_{i j}=(i j)$. For each vertex $V_{i}$, let $R\left(V_{i}, 2 \pi / 3\right)$ denote the rotation matrix around the line through $V_{i}$ and $O$ by angle $2 \pi / 3$. Then the action $R\left(V_{i}, 2 \pi / 3\right)$ conserves $\Omega$ invariant. Observe that $R\left(V_{1}, 2 \pi / 3\right)$ leaves $V_{1}$ invariant and maps $V_{2}$ to $V_{3}, V_{3}$ to $V_{4}$ and $V_{4}$ to $V_{2}$. Thus we write $R\left(V_{1}, 2 \pi / 3\right)=(234) \in S_{4}$. Let $R\left(V_{i j}, \pi\right)$ be the rotation matrix around the line through $V_{i j}$ and $O$ by angle $\pi$. Note that 
the line through $V_{12}$ and $O$ goes through $V_{34}$ also. Then we write

$$
R\left(V_{12}, \pi\right)=R\left(V_{34}, \pi\right)=(12)(34) \in S_{4} .
$$

All subgroups $G_{1}$ through $G_{11}$ have orthogonal representations by using $L_{i j}$, $R\left(V_{i j}, \pi\right)$ and $R\left(V_{i}, 2 j \pi / 3\right)$.

Recall that two solutions $u$ and $v$ are said to be equivalent if $u(x)=v(g x)$ with some $g \in O(N)$. If $H$ and $K$ are subgroups of $O(\Omega)$ and they are conjugate, then any $H$ invariant solution is equivalent to a certain $K$ invariant solution. Indeed, let $K=g^{-1} H g$ with some $g \in O(\Omega)$ and let $u(x)$ be an $H$ invariant solution. Putting $v(x):=u(g x)$, we have

$$
v\left(g^{-1} h g x\right)=u(h g x)=u(g x)=v(x) \quad \text { for } h \in H,
$$

which shows that $v(x)$ is $K$ invariant. Accordingly, the $H$ invariant solution $u$ is equivalent to the $g^{-1} H g$ invariant solution $v$. To get non-equivalent solutions, it is enough to consider $G_{1}$ through $G_{11}$ only and we ignore conjugate subgroups.

Let $u_{i}$ be a $G_{i}$ invariant least energy solution. We shall show that $u_{1}, u_{6}$ and $u_{11}$ have different energy. Observe that $G_{1} \subset G_{6} \subset G_{11}$. Since $\Gamma$ defined by (2.2) depends on $\Omega$ and $G_{i}$, we write it as $\Gamma\left(G_{i}\right)$. Recall that $V_{i j}$ denotes the middle point of the edge connecting $V_{i}$ with $V_{j}$. It is easy to verify that $\operatorname{Fix}\left(G_{6}\right)$ is the line through the points $V_{12}$ and $V_{34}$. The farthest points in $\bar{\Omega}$ from this line are all vertices $V_{1}, V_{2}, V_{3}$ and $V_{4}$, i.e.

$$
\Gamma\left(G_{6}\right)=\left\{V_{1}, V_{2}, V_{3}, V_{4}\right\} .
$$

Then we see that $G_{1}(x) \varsubsetneqq G_{6}(x)$ for $x \in \Gamma\left(G_{6}\right)$. Therefore if $\lambda>0$ is large enough or if $A_{0}$ is sufficiently close to $A$, then it holds that $R\left(u_{1}\right)<R\left(u_{6}\right)$. Since Fix $\left(G_{11}\right)$ consists only of the center $O$ of the tetrahedron, $\Gamma\left(G_{11}\right)$ is the set of all vertices, i.e.

$$
\Gamma\left(G_{11}\right)=\left\{V_{1}, V_{2}, V_{3}, V_{4}\right\} .
$$

We easily compute that $G_{6}(x) \varsubsetneqq G_{11}(x)$ for $x \in \Gamma\left(G_{11}\right)$. Thus $R\left(u_{6}\right)<R\left(u_{11}\right)$. Therefore $u_{1}, u_{6}$ and $u_{11}$ have different energies and hence they are not equivalent.

As long as we use Theorem 2.1 only, we cannot find more solutions. We shall show it. Recall that $\Gamma\left(G_{6}\right)$ is given by (3.2). Since $G_{2} \subset G_{6}$ but $G_{2}\left(V_{1}\right)=$ $G_{6}\left(V_{1}\right)=\left\{V_{1}, V_{2}\right\}$, our theorem does not ensure that $R\left(u_{2}\right)<R\left(u_{6}\right)$, i.e. we cannot distinguish $u_{2}$ from $u_{6}$. Observe the inclusions

$$
G_{3} \subset G_{6}, \quad G_{5} \subset G_{11}, \quad G_{7} \subset G_{11}, \quad G_{9} \subset G_{11}, \quad G_{10} \subset G_{11} .
$$

For all these pairs $G_{i} \subset G_{j}$, by using (3.2) and (3.3), one can prove that $G_{i}(x)=$ $G_{j}(x)$ at some $x \in \Gamma\left(G_{j}\right)$. Therefore for these pairs $i, j$, it is unclear whether 
$u_{i}$ is not equivalent to $u_{j}$. The groups $G_{4}$ and $G_{8}$ remain. We easily see that $\operatorname{Fix}\left(G_{4}\right)=\operatorname{Fix}\left(G_{8}\right)$ and it is the line through two points $V_{4}$ and $O$. Hence

$$
\Gamma\left(G_{4}\right)=\Gamma\left(G_{8}\right)=\left\{V_{1}, V_{2}, V_{3}\right\} .
$$

Therefore $f(r)$ satisfy neither (A) nor (B)' in Corollary 2.2 because in the present example, $f(r)$ is a radial function and so it takes the same value at all vertices. Consequently, we have at least 3 non-equivalent positive solutions.

\section{Group invariant least energy}

In this section, we shall show some lemmas which will be needed for the proof of the main results. Let $L^{q}(\Omega, G)$ denote the set of $G$ invariant functions in $L^{q}(\Omega)$. Define the $L^{2}(\Omega)$ inner product and the $H_{0}^{1}(\Omega)$ inner product by

$$
(u, v)_{L^{2}}:=\int_{\Omega} u v d x, \quad(u, v)_{H_{0}^{1}}:=\int_{\Omega} \nabla u \nabla v d x .
$$

We define the orthogonal complements of $L^{2}(\Omega, G)$ and $H_{0}^{1}(\Omega, G)$ by

$$
\begin{aligned}
L^{2}(\Omega, G)^{\perp} & :=\left\{u \in L^{2}(\Omega):(u, v)_{L^{2}}=0 \text { for all } v \in L^{2}(\Omega, G)\right\}, \\
H_{0}^{1}(\Omega, G)^{\perp} & :=\left\{u \in H_{0}^{1}(\Omega):(u, v)_{H_{0}^{1}}=0 \text { for all } v \in H_{0}^{1}(\Omega, G)\right\} .
\end{aligned}
$$

The next two lemmas have been proved in our paper [31].

LEMma 4.1 ([31]). The following assertions hold.

(a) $H_{0}^{1}(\Omega, G)^{\perp} \subset L^{2}(\Omega, G)^{\perp}$.

(b) Let $1 \leq p, q \leq \infty$ with $1 / p+1 / q=1$. For $u \in L^{p}(\Omega) \cap L^{2}(\Omega, G)^{\perp}$ and $v \in L^{q}(\Omega, G)$, it holds that

$$
\int_{\Omega} u v d x=0 .
$$

The Rayleigh quotient $R(u)$ belongs to $C^{2}\left(H_{0}^{1}(\Omega)\right)$ in the sense of the Fréchet derivative. The second derivative $R^{\prime \prime}(u) v w$ is a continuous bilinear form of $v$ and $w$. We give the expression of $R^{\prime \prime}(u) w^{2}$ in the next lemma.

Lemma $4.2([30])$. Let $u$ be a positive solution of (1.1). For $w \in H_{0}^{1}(\Omega)$, it holds that

$$
\begin{aligned}
R^{\prime \prime}(u) w^{2}= & 2 \int_{\Omega}|\nabla w|^{2} d x\left(\int_{\Omega}|\nabla u|^{2} d x\right)^{-2 /(p+1)} \\
& +2(p-1)\left(\int_{\Omega} \nabla u \nabla w d x\right)^{2}\left(\int_{\Omega}|\nabla u|^{2} d x\right)^{-(p+3) /(p+1)} \\
& -2 p \int_{\Omega} f u^{p-1} w^{2} d x\left(\int_{\Omega}|\nabla u|^{2} d x\right)^{-2 /(p+1)} .
\end{aligned}
$$

The proofs of the main theorems are based on the next proposition, which ensures that $R_{H}<R_{G}$. Here $R_{H}$ and $R_{G}$ have been defined by (1.3). 
Proposition 4.3. Let $H$ and $G$ be closed subgroups of $O(N)$ such that $H \varsubsetneqq$ G. Let $\Omega$ and $\Omega_{1}$ be $G$ invariant bounded domains in $\mathbb{R}^{N}$ such that $\Omega \subset \Omega_{1}$. Let $u$ be a $G$ invariant least energy solution of (1.1) and $\phi$ be a function satisfying

$$
\begin{gathered}
\phi \in W^{1, \infty}\left(\Omega_{1}\right) \cap H_{0}^{1}\left(\Omega_{1}, G\right)^{\perp} \cap H_{0}^{1}\left(\Omega_{1}, H\right), \\
\int_{\Omega}|\nabla \phi|^{2} u^{2} d x<\frac{p-1}{2(2 p-1)} \int_{\Omega}|\nabla u|^{2} \phi^{2} d x .
\end{gathered}
$$

Put $v:=(1+\varepsilon \phi) u$. Then $R(v)<R(u)$ for $\varepsilon>0$ small enough. Therefore $R_{H}<R_{G}$, and no $H$ invariant least energy solution can be $G$ invariant.

PROOF. This proposition has already been proved in our paper [30], however we give a proof for the reader's convenience. Since $\phi \in W^{1, \infty}\left(\Omega_{1}\right)$ and $u \in$ $H_{0}^{1}(\Omega)$, the functions $\phi u$ and $v$ belong to $H_{0}^{1}(\Omega)$. Since $u$ is a solution of (1.1), $R^{\prime}(u)$ vanishes. By the Taylor theorem, we have

$$
R(v)=R(u)+\frac{\varepsilon^{2}}{2} R^{\prime \prime}(u)(\phi u)^{2}+o\left(\varepsilon^{2}\right),
$$

where $o\left(\varepsilon^{2}\right) / \varepsilon^{2} \rightarrow 0$ as $\varepsilon \rightarrow 0$. Let us show that $R^{\prime \prime}(u)(\phi u)^{2}<0$.

We use the Schwarz inequality to get

$$
2|u \phi \nabla u \nabla \phi| \leq 2|\nabla \phi|^{2} u^{2}+\frac{1}{2}|\nabla u|^{2} \phi^{2}
$$

which with (4.3) leads to

$$
\int_{\Omega}|\nabla \phi|^{2} u^{2} d x-2(p-1) \int_{\Omega} u \phi \nabla u \nabla \phi d x<(p-1) \int_{\Omega}|\nabla u|^{2} \phi^{2} d x .
$$

This inequality is rewritten as

$$
\int_{\Omega}\left(|\nabla u|^{2} \phi^{2}+2 u \phi \nabla u \nabla \phi+|\nabla \phi|^{2} u^{2}\right) d x<p \int_{\Omega}\left(|\nabla u|^{2} \phi^{2}+2 u \phi \nabla u \nabla \phi\right) d x .
$$

On the other hand, multiplying (1.1) by $\phi^{2} u$ and integrating it over $\Omega$, we have

$$
\int_{\Omega}\left(|\nabla u|^{2} \phi^{2}+2 u \phi \nabla u \nabla \phi\right) d x=\int_{\Omega} f u^{p+1} \phi^{2} d x .
$$

Combining two expressions above, we obtain

$$
\int_{\Omega}\left(|\nabla u|^{2} \phi^{2}+2 u \phi \nabla u \nabla \phi+|\nabla \phi|^{2} u^{2}\right) d x<p \int_{\Omega} f u^{p+1} \phi^{2} d x,
$$

or equivalently,

$$
\int_{\Omega}|\nabla(\phi u)|^{2} d x<p \int_{\Omega} f u^{p+1} \phi^{2} d x
$$

Extend $u$ by zero outside $\Omega$. Since $|\nabla u|^{2} \in L^{1}\left(\Omega_{1}, G\right)$ and $\phi$ belongs to $H_{0}^{1}\left(\Omega_{1}, G\right)^{\perp} \cap L^{\infty}\left(\Omega_{1}\right)$, Lemma 4.1 shows that

$$
\int_{\Omega}|\nabla u|^{2} \phi d x=\int_{\Omega_{1}}|\nabla u|^{2} \phi d x=0
$$


By the elliptic regularity theorem, $u$ belongs to $L^{\infty}(\Omega)$. Since $\nabla\left(u^{2}\right)=2 u \nabla u \in$ $L^{2}(\Omega)$, i.e. $u^{2} \in H_{0}^{1}(\Omega, G)$, we have

$$
\int_{\Omega} u \nabla u \nabla \phi d x=\frac{1}{2} \int_{\Omega_{1}} \nabla\left(u^{2}\right) \nabla \phi d x=0 .
$$

From the two identities above, it follows that

$$
\int_{\Omega} \nabla u \nabla(\phi u) d x=0 .
$$

Substituting $w=\phi u$ into (4.1) and using the identity above, we obtain

$$
\begin{aligned}
R^{\prime \prime}(u)(\phi u)^{2}= & 2 \int_{\Omega}|\nabla(\phi u)|^{2} d x\left(\int_{\Omega}|\nabla u|^{2} d x\right)^{-2 /(p+1)} \\
& -2 p \int_{\Omega} f u^{p+1} \phi^{2} d x\left(\int_{\Omega}|\nabla u|^{2} d x\right)^{-2 /(p+1)} .
\end{aligned}
$$

By (4.5), the right hand side is negative and hence $R^{\prime \prime}(u)(\phi u)^{2}<0$. From (4.4), it follows that $R(v)<R(u)$ for $\varepsilon>0$ small enough. Since $v \in H_{0}^{1}(\Omega, H)$, it holds that $R_{H} \leq R(v)<R(u)=R_{G}$. Consequently, no $H$ invariant least energy solution can be $G$ invariant.

\section{Estimate of $G$ invariant solutions}

In this section, we give an a priori $L^{\infty}(\Omega)$ estimate of a $G$ invariant least energy solution. Furthermore, we investigate the $L^{2}(\Omega)$ norm of solutions in detail. If $\Omega$ is a $G$ invariant domain, then $\operatorname{dist}(x, \partial \Omega)$ is a $G$ invariant function. Using this function with the same method as in our paper [29], we have the next result.

Lemma 5.1 ([29, Lemma 4.2]). Suppose that $f(x) \geq 0$, $\not \equiv 0$ in $\Omega$. Let $u$ be a $G$ invariant least energy solution. Then for $N \geq 3$, there exists a constant $C>0$ independent of $u$ and $f$ such that

$$
\|u\|_{\infty} \leq C\|f\|_{\infty}^{\alpha}\left(\int_{\Omega} f(x) \operatorname{dist}(x, \partial \Omega)^{p+1} d x\right)^{\beta}
$$

where $\|\cdot\|_{\infty}$ denotes the $L^{\infty}(\Omega)$ norm and $\alpha$ and $\beta$ are given by

$$
\alpha:=\frac{N-2}{N+2-(N-2) p}, \quad \beta:=\frac{-4}{(p-1)(N+2-(N-2) p)} .
$$

When $N=2$, for any $\theta \in(0,1)$ there exists a $C_{\theta}>0$ independent of $u$ and $f$ such that

$$
\|u\|_{\infty} \leq C_{\theta}\|f\|_{\infty}^{(1-\theta) / \theta(p-1)}\left(\int_{\Omega} f(x) \operatorname{dist}(x, \partial \Omega)^{p+1} d x\right)^{-1 / \theta(p-1)} .
$$


Hereafter we denote $\operatorname{Fix}(G)$ by $F$. When $n:=\operatorname{dim} F \geq 1$, we can assume without loss of generality that

$$
F=\left\{\left(x_{1}, \ldots, x_{n}, 0, \ldots, 0\right): x_{i} \in \mathbb{R}\right\} .
$$

Indeed, choose an orthogonal matrix $h$ satisfying

$$
h(F)=\left\{\left(x_{1}, \ldots, x_{n}, 0, \ldots, 0\right): x_{i} \in \mathbb{R}\right\},
$$

and put $\widetilde{u}(x):=u\left(h^{-1} x\right), \widetilde{f}(x):=f\left(h^{-1} x\right)$ and $\widetilde{\Omega}:=h(\Omega)$. Then (1.1) is rewritten as

$$
-\Delta \widetilde{u}=\widetilde{f}(x) \widetilde{u}^{p}, \quad \widetilde{u}>0 \quad \text { in } \widetilde{\Omega}, \quad \widetilde{u}=0 \quad \text { on } \partial \widetilde{\Omega} .
$$

Put $\widetilde{G}:=h G h^{-1}$. Then $u$ is a $G$ invariant solution of (1.1) if and only if $\widetilde{u}$ is a $\widetilde{G}$ invariant solution of the equation above. Furthermore, it holds that $\operatorname{Fix}(\widetilde{G})=h(\operatorname{Fix}(G))$. Therefore we can assume either (a) or (b) below.

(a) $F=\left\{\left(x_{1}, \ldots, x_{n}, 0, \ldots, 0\right): x_{i} \in \mathbb{R}\right\}$ when $\operatorname{dim} F=n \geq 1$.

(b) $F=\{0\}$ when $\operatorname{dim} F=0$.

Some lemmas below have already been proved in our paper [31], however it has not been published yet. Therefore we shall prove them for the reader's convenience.

Lemma 5.2 ([31]). If $\operatorname{dim} F=N-1$, then $G$ takes the form

$$
G=\left\{\left(\begin{array}{ll}
I^{\prime} & 0 \\
0 & 1
\end{array}\right),\left(\begin{array}{rr}
I^{\prime} & 0 \\
0 & -1
\end{array}\right)\right\},
$$

where $I^{\prime}$ denotes the $(N-1) \times(N-1)$ unit matrix. Therefore any $G$ invariant function $u$ is even with respect to $x_{N}$, i.e.

$$
u\left(x_{1}, \ldots, x_{N-1}, x_{N}\right)=u\left(x_{1}, \ldots, x_{N-1},-x_{N}\right) .
$$

Proof. Since $\operatorname{dim} F=N-1$, it holds that

$$
F=\operatorname{Fix}(G)=\left\{\left(x_{1}, \ldots, x_{N-1}, 0\right): x_{i} \in \mathbb{R}\right\},
$$

which implies that $g x=x$ for all $x=\left(x_{1}, \ldots, x_{N-1}, 0\right)$ and $g \in G$. Hence each $g \in G$ has the form

$$
g=\left(\begin{array}{cc}
I^{\prime} & * \\
0 & *
\end{array}\right) .
$$

Since $g$ is orthogonal, it is equal to

$$
\left(\begin{array}{ll}
I^{\prime} & 0 \\
0 & 1
\end{array}\right) \quad \text { or } \quad\left(\begin{array}{rr}
I^{\prime} & 0 \\
0 & -1
\end{array}\right) \text {. }
$$

Since $\operatorname{dim} F=N-1$, it holds that $\{I\} \varsubsetneqq G$. Therefore $G$ takes the form of $(5.2)$. 
By (a) before Lemma 5.2, the orthogonal complement $F^{\perp}$ of $F$ is written as

$$
F^{\perp}:=\left\{\left(0, \ldots, 0, x_{n+1}, \ldots, x_{N}\right): x_{i} \in \mathbb{R}(1 \leq i \leq n)\right\} .
$$

Any point $x \in \mathbb{R}^{N}$ is decomposed into $x=x^{\prime}+x^{\prime \prime}$ with $x^{\prime} \in F$ and $x^{\prime \prime} \in F^{\perp}$. Moreover, in the space $F^{\perp}$, we introduce polar coordinates

$$
x^{\prime \prime}=r \sigma, \quad \text { where } r=\left|x^{\prime \prime}\right|, \quad \sigma=x^{\prime \prime} /\left|x^{\prime \prime}\right| \in S^{N-n-1} .
$$

Here $S^{N-n-1}$ denotes the unit sphere in $F^{\perp}$. Then any function $u(x)$ is represented as $u(x)=u\left(x^{\prime}, x^{\prime \prime}\right)=u\left(x^{\prime}, \sigma, r\right)$. For any $u \in H_{0}^{1}(\Omega)$, we extend $u$ by 0 outside $\Omega$. Then $u \in H^{1}\left(\mathbb{R}^{N}\right)$. For $r>0$, we define

$$
F_{r}:=\left\{x \in \mathbb{R}^{N}: \operatorname{dist}(x, F)=r\right\}=\left\{x^{\prime}+x^{\prime \prime}:\left|x^{\prime \prime}\right|=r\right\} .
$$

Definition 5.3. We put $n:=\operatorname{dim} F$. For a $G$ invariant positive solution $u$, we define

$$
U(r):=\frac{1}{r^{N-n-1}} \int_{F_{r}} u(x)^{2} d s_{r},
$$

where $d s_{r}$ is the standard measure on $F_{r}$, i.e.,

$$
d s_{r}=r^{N-n-1} d x^{\prime} d \sigma=r^{N-n-1} d x_{1} \ldots d x_{n} d \sigma .
$$

Here $d \sigma$ denotes the usual measure on the unit sphere $S^{N-n-1}$ in $F^{\perp}$.

We rewrite $U$ in the following form.

(i) If $1 \leq n \leq N-2$, then

$$
U(r)=\int_{S^{N-n-1}}\left(\int_{\mathbb{R}^{n}} u\left(x^{\prime}, \sigma, r\right)^{2} d x^{\prime}\right) d \sigma .
$$

(ii) If $n=0$, then $F=\{0\}, F^{\perp}=\mathbb{R}^{N}$ and we have

$$
U(r)=\int_{S^{N-1}} u(\sigma, r)^{2} d \sigma .
$$

(iii) If $n=N-1, F_{r}$ consists of two hyperplanes parallel to $F$. By (5.3), we get

$$
\begin{aligned}
U(r) & =\int_{\mathbb{R}^{N-1}} u\left(x^{\prime}, r\right)^{2} d x^{\prime}+\int_{\mathbb{R}^{N-1}} u\left(x^{\prime},-r\right)^{2} d x^{\prime} \\
& =2 \int_{\mathbb{R}^{N-1}} u\left(x^{\prime}, r\right)^{2} d x^{\prime} .
\end{aligned}
$$

For $a>0$, we define the weighted Lebesgue and Sobolev spaces:

$$
\begin{aligned}
L^{1}\left(0, \infty ; r^{a}\right) & :=\left\{v: \int_{0}^{\infty}|v(r)| r^{a} d r<\infty\right\}, \\
W^{1,1}\left(0, \infty ; r^{a}\right) & :=\left\{v: \int_{0}^{\infty}\left(|v(r)|+\left|v_{r}(r)\right|\right) r^{a} d r<\infty\right\},
\end{aligned}
$$

where $v_{r}(r)$ denotes the derivative of $v(r)$. 
Lemma 5.4 ([31]). Let u be a $G$ invariant positive solution. Then the following assertions hold.

(a) $U$ belongs to $L^{\infty}(0, \infty) \cap W^{1,1}\left(0, \infty ; r^{N-n-1}\right) \cap C[0, \infty) \cap C^{1}(0, \infty)$.

(b) When $1 \leq n \leq N-2$,

$$
U_{r}(r)=2 \int_{S^{N-n-1}}\left(\int_{\mathbb{R}^{n}} u u_{r} d x^{\prime}\right) d \sigma
$$

(c) When $n=0$,

$$
U_{r}(r)=2 \int_{S^{N-1}} u u_{r} d \sigma
$$

(d) When $n=N-1$,

$$
U_{r}(r)=4 \int_{\mathbb{R}^{N-1}} u\left(x^{\prime}, r\right) u_{x_{N}}\left(x^{\prime}, r\right) d x^{\prime} .
$$

(e) For any $0 \leq n \leq N-1$ and $0<s<t<A$, we have

$$
t^{N-n-1} U_{r}(t)-s^{N-n-1} U_{r}(s)=2 \int_{\Omega\left(s<\left|x^{\prime \prime}\right|<t\right)}\left(|\nabla u|^{2}-f u^{p+1}\right) d x .
$$

Proof. It is easy to verify (a)-(d). We shall show (e). Let $0<s<t<A$ and put $D:=\Omega\left(s<\left|x^{\prime \prime}\right|<t\right)=\left\{x^{\prime}+x^{\prime \prime} \in \Omega: s<\left|x^{\prime \prime}\right|<t\right\}$. Multiplying (1.1) by $u$ and integrating it over $D$, we have

$$
\int_{D}\left(|\nabla u|^{2}-f u^{p+1}\right) d x=\int_{\partial D} \frac{\partial u}{\partial n} u d \mu,
$$

where $d \mu$ is the standard measure on $\partial D$ and $\partial u / \partial n$ denotes the outward normal derivative. We divide $\partial D$ into $\partial D=S \cup \Omega\left(\left|x^{\prime \prime}\right|=s\right) \cup \Omega\left(\left|x^{\prime \prime}\right|=t\right)$, where $S:=\partial \Omega \cap\left\{x^{\prime}+x^{\prime \prime}: s<\left|x^{\prime \prime}\right|<t\right\}, \Omega\left(\left|x^{\prime \prime}\right|=r\right):=\left\{x^{\prime}+x^{\prime \prime} \in \Omega:\left|x^{\prime \prime}\right|=r\right\}$ with $r=s, t$.

Let $n \leq N-2$. Note that $u=0$ on $S$. Since $\partial / \partial n$ is the outward normal derivative on $\partial D$, it is computed as

$$
\frac{\partial u}{\partial n}=\frac{\partial u}{\partial r} \quad \text { on } \Omega\left(\left|x^{\prime \prime}\right|=t\right), \quad \frac{\partial u}{\partial n}=-\frac{\partial u}{\partial r} \quad \text { on } \Omega\left(\left|x^{\prime \prime}\right|=s\right) .
$$

Note that $d \mu=r^{N-n-1} d x^{\prime} d \sigma$ on $\Omega\left(\left|x^{\prime \prime}\right|=r\right)$, where $d \sigma$ is a standard measure on $S^{N-n-1}$. Using (5.8) or (5.9), we obtain

$$
\begin{aligned}
\int_{\partial D} \frac{\partial u}{\partial n} u d \mu= & \int_{\Omega\left(\left|x^{\prime \prime}\right|=t\right)} \frac{\partial u}{\partial r} u d \mu-\int_{\Omega\left(\left|x^{\prime \prime}\right|=s\right)} \frac{\partial u}{\partial r} u d \mu \\
= & t^{N-n-1} \int_{\Omega\left(\left|x^{\prime \prime}\right|=t\right)} \frac{\partial u}{\partial r} u d x^{\prime} d \sigma \\
& -s^{N-n-1} \int_{\Omega\left(\left|x^{\prime \prime}\right|=s\right)} \frac{\partial u}{\partial r} u d x^{\prime} d \sigma \\
= & t^{N-n-1} U_{r}(t) / 2-s^{N-n-1} U_{r}(s) / 2 .
\end{aligned}
$$


Let $n=N-1$. Then $\Omega\left(\left|x^{\prime \prime}\right|=r\right)=\left\{\left(x^{\prime}, x_{N}\right) \in \Omega: x_{N}=r,-r\right\}$. Since $u\left(x^{\prime}, x_{N}\right)$ is even in $x_{N}$ by Lemma 5.2, we use (5.10) to get

$$
\int_{\Omega\left(\left|x^{\prime \prime}\right|=t\right)} \frac{\partial u}{\partial r} u d x^{\prime}=2 \int_{\mathbb{R}^{N-1}} \frac{\partial u}{\partial x_{N}}\left(x^{\prime}, t\right) u\left(x^{\prime}, t\right) d x^{\prime}=U_{r}(t) / 2 .
$$

Therefore (5.13) is valid for $n=N-1$ also. Substituting (5.13) into (5.12), we obtain (5.11). The proof is complete.

We study the relation between the $L^{\infty}$ norm of $U(r)$ and the $L^{2}$ norm of $\nabla u$.

Lemma 5.5. ([31]) Let $u(x)$ and $U(r)$ be as in Lemma 5.4. Put $m:=N-n-1$. Then for any $a \in(0, A)$, it holds that

$$
\max _{a \leq r \leq A} U(r) \leq 4(A-a) a^{-m} \int_{\Omega\left(a<\left|x^{\prime \prime}\right|<A\right)}|\nabla u|^{2} d x .
$$

Proof. Let $b$ be a maximum point of $U(r)$ on $[a, A]$. Let $1 \leq n \leq N-2$ and set $D:=S^{m} \times \mathbb{R}^{n}$. Then (5.5) and (5.8) are rewritten as

$$
U(r)=\int_{D} u^{2} d x^{\prime} d \sigma, \quad U_{r}(r)=2 \int_{D} u u_{r} d x^{\prime} d \sigma
$$

Applying the Schwarz inequality to the second equation above, we have

$$
\begin{aligned}
\left|U_{r}(r)\right| & \leq 2\left(\int_{D} u^{2} d x^{\prime} d \sigma\right)^{1 / 2}\left(\int_{D} u_{r}^{2} d x^{\prime} d \sigma\right)^{1 / 2} \\
& \leq 2 U(b)^{1 / 2}\left(\int_{D} u_{r}^{2} d x^{\prime} d \sigma\right)^{1 / 2},
\end{aligned}
$$

for $r \in[a, A]$. Using the inequality above and $U(A)=0$, we get

$$
\begin{aligned}
0 \leq U(b) & \leq \int_{b}^{A}\left|U_{r}(r)\right| d r \\
& \leq\left(\int_{b}^{A}\left|U_{r}(r)\right|^{2} r^{m} d r\right)^{1 / 2}\left(\int_{b}^{A} r^{-m} d r\right)^{1 / 2} \\
& \leq 2\left((A-b) b^{-m}\right)^{1 / 2} U(b)^{1 / 2}\left(\int_{b}^{A} \int_{D} u_{r}^{2} r^{m} d x^{\prime} d \sigma d r\right)^{1 / 2} \\
& \leq 2\left((A-b) b^{-m}\right)^{1 / 2} U(b)^{1 / 2}\|\nabla u\|_{L^{2}\left(\Omega\left(b \leq\left|x^{\prime \prime}\right| \leq A\right)\right) .}
\end{aligned}
$$

Since $a \leq b$, we have

$$
U(b) \leq 4(A-a) a^{-m}\|\nabla u\|_{L^{2}\left(\Omega\left(a \leq\left|x^{\prime \prime}\right| \leq A\right)\right)}^{2} .
$$

This proves (5.14). Let $n=N-1$. Then $m=0$ and

Instead of (5.15), we have

$$
U(r)=2 \int_{\mathbb{R}^{N-1}} u^{2} d x^{\prime}, \quad U_{r}(r)=4 \int_{\mathbb{R}^{N-1}} u u_{x_{N}} d x^{\prime} .
$$

$$
\left|U_{r}(r)\right| \leq(4 / \sqrt{2}) U(b)^{1 / 2}\left(\int_{\mathbb{R}^{N-1}} u_{x_{N}}^{2} d x^{\prime}\right)^{1 / 2} .
$$


In the same way as in (5.16) with the inequality above, we obtain

$$
0 \leq U(b) \leq(4 / \sqrt{2})(A-b)^{1 / 2} U(b)^{1 / 2}\left(\int_{b}^{A} \int_{\mathbb{R}^{N-1}} u_{x_{N}}^{2} d x^{\prime} d r\right)^{1 / 2} .
$$

Note that

$$
\int_{b}^{A} \int_{\mathbb{R}^{N-1}} u_{x_{N}}^{2} d x^{\prime} d r=\|\nabla u\|_{L^{2}(\Omega(b<r<A))}^{2}=(1 / 2)\|\nabla u\|_{L^{2}\left(\Omega\left(b<\left|x_{N}\right|<A\right)\right)}^{2} .
$$

Therefore we obtain (5.16) for $n=N-1$. The method above is still valid for $n=0$ also.

Lemma 5.6 ([31]). If $f(x) \leq 0$ in $\Omega\left(\left|x^{\prime \prime}\right|<a\right)$, then $U(r)$ is nondecreasing in $(0, a)$.

Proof. Multiplying (1.1) by $u$ and integrating it over $\Omega$, we have

$$
\int_{\Omega}\left(|\nabla u|^{2}-f u^{p+1}\right) d x=0 .
$$

We set $m:=N-n-1$. Then (5.11) is reduced to

$$
t^{m} U_{r}(t)-s^{m} U_{r}(s)=2 \int_{\Omega\left(s<\left|x^{\prime \prime}\right|<t\right)}\left(|\nabla u|^{2}-f u^{p+1}\right) d x .
$$

Since $u$ is extended by 0 outside $\Omega$, it belongs to $H_{0}^{1}\left(\mathbb{R}^{N}\right)$. Since $U(t)=0$ for $t \geq A$, we substitute $t=A$ to obtain

$$
-s^{m} U_{r}(s)=2 \int_{\Omega\left(s<\left|x^{\prime \prime}\right|<A\right)}\left(|\nabla u|^{2}-f u^{p+1}\right) d x .
$$

As $s \rightarrow 0$, the right hand side converges to

$$
2 \int_{\Omega}\left(|\nabla u|^{2}-f u^{p+1}\right) d x=0
$$

because of (5.17). Thus $s^{m} U_{r}(s)$ converges to zero. Letting $s \rightarrow 0$ in (5.18), we find

$$
t^{m} U_{r}(t)=2 \int_{\Omega\left(\left|x^{\prime \prime}\right|<t\right)}\left(|\nabla u|^{2}-f u^{p+1}\right) d x \geq 0,
$$

for $0<t<a$. Therefore $U(t)$ is nondecreasing in $(0, a)$.

\section{Proof of the main results}

In this section, we prove the main theorems. Our idea is based on the methods used in our papers [30], [31] and [32]. Since a closed subgroup $G$ of $O(N)$ is a compact Lie group, it has a unique Haar measure $d g$. It is a positive Lebesgue measure which satisfies

$$
\begin{gathered}
\int_{G} f(h g) d g=\int_{G} f(g h) d g=\int_{G} f\left(g^{-1}\right) d g=\int_{G} f(g) d g, \\
\int_{G} f(g) d g>0 \quad \text { if } f \geq 0, \not \equiv 0
\end{gathered}
$$




$$
\int_{G} 1 d g=1
$$

for any $h \in G$ and any real valued integrable function $f$ on $G$ (see [47] or [42, Chapter 2] for more details of the Haar measure).

Let $M(N)$ denote a linear space consisting of all $N \times N$ real matrices, which is equipped with the norm

$$
\|g\|:=\max _{|x| \leq 1}|g x| \quad \text { for } g \in M(N) .
$$

Here $|x|$ is the usual Euclidean norm in $\mathbb{R}^{N}$. For $g_{0} \in G$ and $r>0$, we define a ball $B\left(g_{0}, r ; G\right)$ in $G$ by

$$
B\left(g_{0}, r ; G\right):=\left\{g \in G:\left\|g-g_{0}\right\|<r\right\} .
$$

Then the volume of $B\left(g_{0}, r ; G\right)$ is defined by

$$
\left|B\left(g_{0}, r ; G\right)\right|:=\int_{B\left(g_{0}, r ; G\right)} 1 d g .
$$

The invariance of the Haar measure implies the next lemma.

Lemma 6.1 ([32]). Let $G$ be a closed subgroup of $O(N)$. Then the volume $\left|B\left(g_{0}, r ; G\right)\right|$ is independent of $g_{0} \in G$ but depends only on $r$.

Proof. Put $\chi(g):=1$ if $g \in B\left(g_{0}, r ; G\right)$ and $\chi(g):=0$ if $g \notin B\left(g_{0}, r ; G\right)$. Then

$$
\left|B\left(g_{0}, r ; G\right)\right|=\int_{G} \chi(g) d g .
$$

Let $g, g_{1} \in G$. Since $g, g_{1}, g_{0}$ are isometric in $\mathbb{R}^{N}$, it holds that

$$
\left\|g_{0} g_{1}^{-1} g-g_{0}\right\|=\max _{|x| \leq 1}\left|g_{0} g_{1}^{-1} g x-g_{0} x\right|=\max _{|x| \leq 1}\left|g x-g_{1} x\right|=\left\|g-g_{1}\right\| .
$$

Therefore $\chi\left(g_{0} g_{1}^{-1} g\right)=1$ if $\left\|g-g_{1}\right\|<r$ and $\chi\left(g_{0} g_{1}^{-1} g\right)=0$ if $\left\|g-g_{1}\right\| \geq r$. By the invariance of the Haar measure, we get

$$
\int_{G} \chi(g) d g=\int_{G} \chi\left(g_{0} g_{1}^{-1} g\right) d g=\int_{B\left(g_{1}, r ; G\right)} 1 d g .
$$

For closed subgroups $G$ and $H$ of $O(N)$, we define

$$
Q(x, g):=\min _{h \in H}|g x-h x|, \quad P(x):=\max _{g \in G} Q(x, g) .
$$

Lemma 6.2. ([32]) It holds that $|P(x)-P(y)| \leq 2|x-y|$ for $x, y \in \mathbb{R}^{N}$.

Proof. Let $x, y \in \mathbb{R}^{N}, g \in G$ and $h \in H$. Since $g$ and $h$ are orthogonal matrices, we have

$$
Q(x, g) \leq|g x-h x| \leq|g x-g y|+|g y-h y|+|h y-h x|=2|x-y|+|g y-h y| .
$$


Taking the minimum on $h \in H$, we obtain $Q(x, g) \leq 2|x-y|+Q(y, g)$. By the definition of $P, Q(x, g) \leq 2|x-y|+P(y)$. Taking the maximum on $g \in G$, we get $P(x) \leq 2|x-y|+P(y)$. Exchanging $x$ with $y$ implies the conclusion.

By Lemma 6.2, $P(x)$ is continuous. Assumption (2.3) implies that $P(x)>0$ for $x \in \Gamma$, and so the minimum of $P(x)$ on $\Gamma$ is positive. We define

$$
\delta:=(1 / 8) \min _{\Gamma} P(x)>0 .
$$

We choose $A_{1} \in(0, A)$ slightly less than $A$ such that

$$
P(x)>4 \delta \quad \text { for } x \in \Omega\left(A_{1} \leq\left|x^{\prime \prime}\right| \leq A\right) .
$$

Define

$$
\Omega_{0}:=\Omega\left(A_{1}<\left|x^{\prime \prime}\right|<A\right) .
$$

By (6.4) and (6.6), for any $x \in \bar{\Omega}_{0}$ there is a $g \in G$ such that

$$
|g x-h x|>4 \delta>0 \text { for all } h \in H .
$$

Let $\Phi \in C_{0}^{\infty}\left(\mathbb{R}^{N}\right)$ be a radial function such that $0 \leq \Phi(|x|) \leq 1$ in $\mathbb{R}^{N}$ and $\Phi(|x|)=1$ when $|x| \leq \delta$ and $\Phi(|x|)=0$ when $|x| \geq 2 \delta$. We denote the Haar measures on $H$ and $G$ by $d h$ and $d g$, respectively. We define

$$
\phi(x):=\int_{G} \Phi\left(\left|x-g x_{0}\right|\right) d g-\int_{H} \Phi\left(\left|x-h x_{0}\right|\right) d h,
$$

where $x_{0} \in \bar{\Omega}$ will be determined later on. Moreover, we define

$$
\operatorname{dist}(x, \Omega):=\inf \{|x-y|: y \in \Omega\}, \quad \Omega_{1}:=\left\{x \in \mathbb{R}^{N}: \operatorname{dist}(x, \Omega)<2 \delta\right\} .
$$

Clearly $\Omega \subset \Omega_{1}$. The function $\phi$ defined by (6.9) satisfies

Lemma 6.3. $\phi \in C_{0}^{\infty}\left(\Omega_{1}\right) \cap H_{0}^{1}\left(\Omega_{1}, G\right)^{\perp} \cap H_{0}^{1}\left(\Omega_{1}, H\right)$.

Proof. Since $x_{0} \in \bar{\Omega}$ and $\Phi(|x|)=0$ for $|x| \geq 2 \delta$, the support of $\phi$ is in $\Omega_{1}$. Give $\psi \in H_{0}^{1}\left(\Omega_{1}, G\right)$ arbitrarily. Let $g \in G$. Since $\psi$ is $G$ invariant, the change of variables $x=g y$ yields

$$
\begin{aligned}
\int_{\left|x-g x_{0}\right|<2 \delta} \nabla \Phi\left(\left|x-g x_{0}\right|\right) \nabla \psi d x & =\int_{\left|g y-g x_{0}\right|<2 \delta} \nabla \Phi\left(\left|g y-g x_{0}\right|\right) \nabla \psi(g y) d y \\
& =\int_{B\left(x_{0}, 2 \delta\right)} \nabla \Phi\left(\left|y-x_{0}\right|\right) \nabla \psi(y) d y .
\end{aligned}
$$

Since the support of $\Phi\left(\left|x-g x_{0}\right|\right)$ is in the ball $B\left(g x_{0}, 2 \delta\right)$, the identity above is rewritten as

$$
\int_{\Omega_{1}} \nabla \Phi\left(\left|x-g x_{0}\right|\right) \nabla \psi d x=\int_{\Omega_{1}} \nabla \Phi\left(\left|x-x_{0}\right|\right) \nabla \psi(x) d x,
$$


for any $g \in G$. Using this relation with the Fubini theorem and employing (6.2), we obtain

$$
\begin{aligned}
\int_{\Omega_{1}} \nabla\left(\int_{G} \Phi\left(\left|x-g x_{0}\right|\right) d g\right) \nabla \psi d x & =\int_{G}\left(\int_{\Omega_{1}} \nabla \Phi\left(\left|x-g x_{0}\right|\right) \nabla \psi d x\right) d g \\
& =\int_{\Omega_{1}} \nabla \Phi\left(\left|x-x_{0}\right|\right) \nabla \psi d x .
\end{aligned}
$$

Similarly, we get

$$
\int_{\Omega_{1}} \nabla\left(\int_{H} \Phi\left(\left|x-h x_{0}\right|\right) d h\right) \nabla \psi d x=\int_{\Omega_{1}} \nabla \Phi\left(\left|x-x_{0}\right|\right) \nabla \psi d x .
$$

Subtracting the latter equation from the former one, we obtain

$$
\int_{\Omega_{1}} \nabla \phi \nabla \psi d x=0
$$

which means that $\phi \in H_{0}^{1}\left(\Omega_{1}, G\right)^{\perp}$.

We shall show that $\phi \in H_{0}^{1}\left(\Omega_{1}, H\right)$. Give $k \in H$ arbitrarily. By the invariance of the Haar measure, we have

$$
\begin{aligned}
\phi(k x) & =\int_{G} \Phi\left(\left|k x-g x_{0}\right|\right) d g-\int_{H} \Phi\left(\left|k x-h x_{0}\right|\right) d h \\
& =\int_{G} \Phi\left(\left|x-k^{-1} g x_{0}\right|\right) d g-\int_{H} \Phi\left(\left|x-k^{-1} h x_{0}\right|\right) d h \\
& =\int_{G} \Phi\left(\left|x-g x_{0}\right|\right) d g-\int_{H} \Phi\left(\left|x-h x_{0}\right|\right) d h=\phi(x) .
\end{aligned}
$$

Thus $\phi \in H_{0}^{1}\left(\Omega_{1}, H\right)$ and the proof is complete.

Hereafter $B(x, r)$ denotes the ball in $\mathbb{R}^{N}$ which is centered at $x$ with radius $r$ and $|B(x, r)|$ stands for its Lebesgue measure. Recall that $B(g, r ; G)$ defined by (6.3) denotes a ball in $G$ and its volume $|B(g, r ; G)|$ depends only on $r$. Since $\Omega$ is bounded, we choose a constant $M>0$ such that $|x| \leq M$ for $x \in \Omega$. Put $\nu:=\delta /(2 M)$ and define $c_{\nu}:=|B(g, \nu ; G)|$. Let $\Omega_{0}$ be defined by (6.7). Then we have

Lemma 6.4. For any $x_{0} \in \bar{\Omega}_{0}$, there exists a $g_{0} \in G$ such that

$$
\phi(x) \geq c_{\nu}>0 \quad \text { for } x \in B\left(g_{0} x_{0}, \delta / 2\right) .
$$

In particular, $\phi \not \equiv 0$ in $\Omega$.

Proof. Let $x_{0} \in \bar{\Omega}_{0}$. By (6.8), we choose a $g_{0} \in G$ such that

$$
\left|g_{0} x_{0}-h x_{0}\right|>4 \delta \text { for all } h \in H .
$$

Then $B\left(g_{0} x_{0}, 2 \delta\right) \cap B\left(h x_{0}, 2 \delta\right)=\emptyset$ for $h \in H$, which shows that

$$
\Phi\left(\left|x-h x_{0}\right|\right)=0 \quad \text { for } x \in B\left(g_{0} x_{0}, 2 \delta\right), h \in H .
$$


The definition of $\phi$ implies

$$
\phi(x)=\int_{G} \Phi\left(\left|x-g x_{0}\right|\right) d g \quad \text { for } x \in B\left(g_{0} x_{0}, 2 \delta\right) .
$$

For $x \in B\left(g_{0} x_{0}, \delta / 2\right)$ and $g \in B\left(g_{0}, \nu ; G\right)$, it holds that

$$
\left|x-g x_{0}\right| \leq\left|x-g_{0} x_{0}\right|+\left|g_{0} x_{0}-g x_{0}\right|<\delta / 2+\nu M=\delta .
$$

Hence we have

$$
\Phi\left(\left|x-g x_{0}\right|\right)=1 \quad \text { for } x \in B\left(g_{0} x_{0}, \delta / 2\right), \quad g \in B\left(g_{0}, \nu ; G\right) .
$$

Consequently, (6.11) implies

$$
\phi(x) \geq \int_{B\left(g_{0}, \nu ; G\right)} \Phi\left(\left|x-g x_{0}\right|\right) d g=\left|B\left(g_{0}, \nu ; G\right)\right|=c_{\nu}>0,
$$

for $x \in B\left(g_{0} x_{0}, \delta / 2\right)$. This completes the proof.

For $\Omega_{0}$ defined by (6.7), we determine $k \in \mathbb{N}$ by the smallest positive integer such that

$$
\bar{\Omega}_{0} \subset \bigcup_{i=1}^{k} B\left(y_{i}, \delta / 2\right)
$$

with some $y_{1}, \ldots, y_{k} \in \bar{\Omega}_{0}$.

Lemma 6.5. Let u be a $G$ invariant least energy solution and $k$ be as above. Then there exist $x_{0} \in \bar{\Omega}_{0}$ and $g_{0} \in G$ such that

$$
\int_{\Omega_{0}}|\nabla u|^{2} d x \leq k c_{\nu}^{-2} \int_{B\left(g_{0} x_{0}, \delta / 2\right)}|\nabla u|^{2} \phi^{2} d x .
$$

Proof. Choose $x_{0} \in \bar{\Omega}_{0}$ which satisfies

$$
\max _{y \in \overline{\Omega_{0}}} \int_{B(y, \delta / 2)}|\nabla u|^{2} d x=\int_{B\left(x_{0}, \delta / 2\right)}|\nabla u|^{2} d x .
$$

By (6.12), we have

$$
\int_{\Omega_{0}}|\nabla u|^{2} d x \leq k \int_{B\left(x_{0}, \delta / 2\right)}|\nabla u|^{2} d x .
$$

By Lemma 6.4, we choose $g_{0} \in G$ satisfying (6.10). Since $u$ is $G$ invariant, we have

$$
\int_{\Omega_{0}}|\nabla u|^{2} d x \leq k \int_{B\left(g_{0} x_{0}, \delta / 2\right)}|\nabla u|^{2} d x \leq k c_{\nu}^{-2} \int_{B\left(g_{0} x_{0}, \delta / 2\right)}|\nabla u|^{2} \phi^{2} d x .
$$

We give the definition of $\phi(x)$ strictly. First, we define $\delta>0$ by (6.5). Second, we define $A_{1}$ by (6.6) and then $\Omega_{0}$ by (6.7). Third, we fix $x_{0}$ by Lemma 6.5 . Last, $\phi(x)$ is well defined by (6.9). 
Lemma 6.6. Let $u$ be a $G$ invariant least energy solution. Let $g_{0}, x_{0}, k, c_{\nu}$ be as in Lemma 6.5 and put $m:=N-n-1$. Then for any $a \in\left[A_{1}, A\right]$, we have

$$
\max _{a \leq r \leq A} U(r) \leq 4 k(A-a) a^{-m} c_{\nu}^{-2} \int_{B\left(g_{0} x_{0}, \delta / 2\right)}|\nabla u|^{2} \phi^{2} d x .
$$

Proof. Since $\Omega_{0}=\Omega\left(A_{1}<\left|x^{\prime \prime}\right|<A\right)$, we use Lemma 6.5 to get

$$
\int_{\Omega\left(a<\left|x^{\prime \prime}\right|<A\right)}|\nabla u|^{2} d x \leq \int_{\Omega\left(A_{1}<\left|x^{\prime \prime}\right|<A\right)}|\nabla u|^{2} d x \leq k c_{\nu}^{-2} \int_{B\left(g_{0} x_{0}, \delta / 2\right)}|\nabla u|^{2} \phi^{2} d x \text {. }
$$

Combining the inequality above with (5.14), we get the conclusion.

We note that $\delta, \Omega_{0}, c_{\nu}, k$ and $m$ depend only on $G, H, \Omega$ and not on $f(x)$. Choose $A_{0} \in\left(A_{1}, A\right)$ sufficiently close to $A$ such that

$$
32 k(m+1)^{-1} A^{m+1}\left(A-A_{0}\right) A_{0}^{-m} c_{\nu}^{-2}\|\nabla \Phi\|_{\infty}^{2}<\frac{p-1}{2(2 p-1)} .
$$

Observe that $\Phi$ depends only on $\delta$, which is determined by $G, H$ and $\Omega$. Consequently, $A_{0}$ depends only on $G, H, \Omega$ and does not on $u(x)$ and $f(x)$. By (6.9), we have

$$
\|\nabla \phi\|_{L^{\infty}\left(\mathbb{R}^{N}\right)} \leq 2\|\nabla \Phi\|_{L^{\infty}\left(\mathbb{R}^{N}\right)} .
$$

Combining two inequalities above, we obtain

$$
8 k(m+1)^{-1} A^{m+1}\left(A-A_{0}\right) A_{0}^{-m} c_{\nu}^{-2}\|\nabla \phi\|_{\infty}^{2}<\frac{p-1}{2(2 p-1)} .
$$

To prove Theorem 2.1, it is enough to show that $\phi$ satisfies (4.2) and (4.3). Lemma 6.3 proves (4.2). Let us verify (4.3).

LEMma 6.7. Let $u$ be a $G$ invariant least energy solution. If the maximum of $U(r)$ on $[0, A]$ is achieved at a point in $\left[A_{0}, A\right]$, then (4.3) holds.

Proof. Denote the maximum point of $U(r)$ on $\left[A_{0}, A\right]$ by $a$. By assumption,

$$
\max _{0 \leq r \leq A} U(r)=\max _{A_{0} \leq r \leq A} U(r)=U(a) .
$$

By Lemma 6.6, we have

$$
U(a)=\max _{A_{0} \leq r \leq A} U(r) \leq 4 k\left(A-A_{0}\right) A_{0}^{-m} c_{\nu}^{-2} \int_{B\left(g_{0} x_{0}, \delta / 2\right)}|\nabla u|^{2} \phi^{2} d x .
$$

Put $D:=S^{m} \times \mathbb{R}^{N}$. Then it holds clearly that

$$
\int_{\Omega} u^{2} d x=\int_{0}^{A}\left(\int_{D} u^{2} r^{m} d x^{\prime} d \sigma\right) d r \leq(m+1)^{-1} A^{m+1} U(a)
$$

which implies

$$
\int_{\Omega}|\nabla \phi|^{2} u^{2} d x \leq\|\nabla \phi\|_{\infty}^{2} \int_{\Omega} u^{2} d x \leq\|\nabla \phi\|_{\infty}^{2}(m+1)^{-1} A^{m+1} U(a) .
$$

Substituting (6.15) into the right hand side of the inequality above and using (6.14), we obtain (4.3). 
The next lemma shows that if assumption (A) before Theorem 2.1 is valid, then (4.3) holds.

LEMma 6.8. Let $u$ be a $G$ invariant least energy solution. If $f(x) \leq 0$ in $\left.\Omega\left(\left|x^{\prime \prime}\right|<A_{0}\right)\right)$, then (4.3) holds.

Proof. Under the assumption of the lemma, $U(r)$ is nondecreasing in $\left(0, A_{0}\right)$ by Lemma 5.6. Thus the maximum of $U(r)$ on $[0, A]$ is achieved at a point in $\left[A_{0}, A\right]$. Therefore Lemma 6.7 ensures the conclusion.

By Lemma 6.7, inequality (4.3) holds if the maximum of $U(r)$ on $[0, A]$ is achieved at a point in $\left[A_{0}, A\right]$. In particular, if $f \leq 0$ in $\Omega\left(\left|x^{\prime \prime}\right|<A_{0}\right)$, i.e. assumption (A) is valid, then (4.3) holds. We consider the remained case where assumption (B) holds and the maximum of $U(r)$ on $[0, A]$ is achieved at a point in $\left[0, A_{0}\right)$.

Lemma 6.9. Assume that $f \geq 0, \not \equiv 0$ in $\Omega$. Let $u$ be a $G$ invariant least energy solution. Suppose that the maximum of $U(r)$ on $[0, A]$ is achieved at a point $a \in\left[0, A_{0}\right)$. If $\mu\left(f, A_{0}\right)$ is small enough with $N=3$ or $\mu\left(f, A_{0}, \theta\right)$ is small enough with $N=2$, then (4.3) holds.

Proof. Since $U(r)$ has a maximum at $a, U_{r}(a)$ vanishes when $a>0$. We substitute $s=a$ in (5.18) to get

$$
t^{m} U_{r}(t)=2 \int_{\Omega\left(a<\left|x^{\prime \prime}\right|<t\right)}\left(|\nabla u|^{2}-f u^{p+1}\right) d x .
$$

As proved in Lemma 5.6, $s^{m} U_{r}(s)$ converges to 0 as $s \rightarrow 0$. Hence, even if $a=0$, the identity above holds. We estimate it as

$$
-t^{m} U_{r}(t) \leq 2 \int_{\Omega\left(a<\left|x^{\prime \prime}\right|<t\right)} f u^{p+1} d x=2 \int_{a}^{t}\left(\int_{D} f u^{p+1} r^{m} d x^{\prime} d \sigma\right) d r,
$$

with $D:=S^{m} \times \mathbb{R}^{n}$. For $t \in\left[a, A_{0}\right]$, we compute

$$
\begin{gathered}
\int_{a}^{t}\left(\int_{D} f u^{p+1} r^{m} d x^{\prime} d \sigma\right) d r \\
\leq\|f\|_{L^{\infty}\left(\Omega\left(\left|x^{\prime \prime}\right|<A_{0}\right)\right)}\|u\|_{L^{\infty}(\Omega)}^{p-1} \int_{a}^{t}\left(\int_{D} u^{2} r^{m} d x^{\prime} d \sigma\right) d r \\
\leq t^{m}(t-a)\|f\|_{L^{\infty}\left(\Omega\left(\left|x^{\prime \prime}\right|<A_{0}\right)\right)}\|u\|_{L^{\infty}(\Omega)}^{p-1} U(a)
\end{gathered}
$$

Therefore we have

$$
-U_{r}(t) \leq 2(t-a)\|f\|_{L^{\infty}\left(\Omega\left(\left|x^{\prime \prime}\right|<A_{0}\right)\right)}\|u\|_{L^{\infty}(\Omega)}^{p-1} U(a) .
$$

Integrating both sides over $\left(a, A_{0}\right)$, we obtain

$$
0 \leq U(a)-U\left(A_{0}\right) \leq A_{0}^{2}\|f\|_{L^{\infty}\left(\Omega\left(\left|x^{\prime \prime}\right|<A_{0}\right)\right)}\|u\|_{L^{\infty}(\Omega)}^{p-1} U(a) .
$$

We shall show that $A_{0}^{2}\|f\|_{L^{\infty}\left(\Omega\left(\left|x^{\prime \prime}\right|<A_{0}\right)\right)}\|u\|_{L^{\infty}(\Omega)}^{p-1} \leq 1 / 2$. 
Let $N \geq 3$. Then Lemma 5.1 implies that

$$
\begin{aligned}
& A_{0}^{2}\|f\|_{L^{\infty}\left(\Omega\left(\left|x^{\prime \prime}\right|<A_{0}\right)\right)}\|u\|_{L^{\infty}(\Omega)}^{p-1} \\
& \quad \leq A_{0}^{2} C^{p-1}\|f\|_{L^{\infty}\left(\Omega\left(\left|x^{\prime \prime}\right|<A_{0}\right)\right)}\|f\|_{L^{\infty}(\Omega)}^{\alpha(p-1)}\left(\int_{\Omega} f(x) \operatorname{dist}(x, \partial \Omega)^{p+1} d x\right)^{\beta(p-1)} \\
& \quad=A_{0}^{2} C^{p-1} \mu\left(f, A_{0}\right)^{1 /(N+2-(N-2) p)} \leq 1 / 2,
\end{aligned}
$$

provided that $\mu\left(f, A_{0}\right)$ is small enough. Here $\alpha$ and $\beta$ have been defined by Lemma 5.1 and $C>0$ is independent of $a, f(x)$ and $u(x)$.

Let $N=2$. By Lemma 5.1, we have

$$
\begin{aligned}
& A_{0}^{2}\|f\|_{L^{\infty}\left(\Omega\left(\left|x^{\prime \prime}\right|<A_{0}\right)\right)}\|u\|_{L^{\infty}(\Omega)}^{p-1} \\
& \quad \leq A_{0}^{2}\|f\|_{L^{\infty}\left(\Omega\left(\left|x^{\prime \prime}\right|<A_{0}\right)\right)} C_{\theta}^{p-1}\|f\|_{L^{\infty}(\Omega)}^{(1-\theta) / \theta}\left(\int_{\Omega} f(x) \operatorname{dist}(x, \partial \Omega)^{p+1} d x\right)^{-1 / \theta} \\
& \quad=A_{0}^{2} C_{\theta}^{p-1} \mu\left(f, A_{0}, \theta\right) \leq 1 / 2,
\end{aligned}
$$

provided that $\mu\left(f, A_{0}, \theta\right)$ is small enough. Consequently, if $\mu\left(f, A_{0}\right)$ and $\mu\left(f, A_{0}, \theta\right)$ are small enough, we have

$$
A_{0}^{2}\|f\|_{L^{\infty}\left(\Omega\left(\left|x^{\prime \prime}\right|<A_{0}\right)\right)}\|u\|_{L^{\infty}(\Omega)}^{p-1} \leq 1 / 2 .
$$

This inequality with (6.16) implies that $U(a) \leq 2 U\left(A_{0}\right)$. By Lemma 6.6 and (6.14), we obtain

$$
\begin{aligned}
& \int_{\Omega}|\nabla \phi|^{2} u(x)^{2} d x \leq\|\nabla \phi\|_{\infty}^{2} \int_{0}^{A}\left(\int_{D} u^{2} r^{m} d x^{\prime} d \sigma\right) d r \\
& \leq\|\nabla \phi\|_{\infty}^{2}(m+1)^{-1} A^{m+1} U(a) \leq 2\|\nabla \phi\|_{\infty}^{2}(m+1)^{-1} A^{m+1} U\left(A_{0}\right) \\
& \leq 8 k\left(A-A_{0}\right) A_{0}^{-m} c_{\nu}^{-2}\|\nabla \phi\|_{\infty}^{2}(m+1)^{-1} A^{m+1} \int_{B\left(g_{0} x_{0}, \delta / 2\right)}|\nabla u|^{2} \phi^{2} d x \\
& <\frac{p-1}{2(2 p-1)} \int_{\Omega}|\nabla u|^{2} \phi^{2} d x .
\end{aligned}
$$

We are now in a position to prove Theorem 2.1. Because of Proposition 4.3, it is enough to show (4.3).

Proof of Theorem 2.1. If (A) holds, then Lemma 6.8 ensures (4.3). If (B) holds, then Lemmas 6.7 and 6.9 imply (4.3).

Proof of Corollary 2.2. We shall show that (B)' implies (B). We deal with $N \geq 3$ only because the method below is valid for $N=2$ also. We fix $A_{0}$ so close to $A$ that Theorem 2.1 is valid under assumption (B) with $A_{0}$. Define

$$
M:=\max _{x \in \Gamma} f_{0}(x), \quad m:=\left\|f_{0}\right\|_{L^{\infty}\left(\Omega\left(\left|x^{\prime \prime}\right| \leq A_{0}\right)\right)} .
$$

Since $m<M$ by assumption, we can choose $M_{1} \in(0, M)$ sufficiently close to $M$ such that

$$
m^{N+2-(N-2) p} M^{(N-2)(p-1)} M_{1}^{-4}<1 .
$$


Choose $x_{0} \in \Gamma$ satisfying $f_{0}\left(x_{0}\right)=M$. Take an $\varepsilon>0$ such that $f_{0}(x)>M_{1}$ for $x \in \Omega \cap B\left(x_{0}, \varepsilon\right)$. Then we have

$$
\int_{\Omega} f(x) \operatorname{dist}(x, \partial \Omega)^{p+1} d x \geq \int_{\Omega \cap B\left(x_{0}, \varepsilon\right)} f(x) \operatorname{dist}(x, \partial \Omega)^{p+1} d x \geq C M_{1}^{\lambda},
$$

where $C>0$ is independent of $\lambda$. Therefore we obtain

$$
\mu\left(f, A_{0}\right) \leq C\left(m^{N+2-(N-2) p} M^{(N-2)(p-1)} M_{1}^{-4}\right)^{\lambda} \rightarrow 0 \quad \text { as } \lambda \rightarrow \infty .
$$

Hence (B) holds for $\lambda>0$ large enough and the proof is complete.

We shall show Examples 3.3 and 3.4

Proof of Example 3.3. Let $G_{n}$ be as in (1.5). Then it is easy to see that

$$
g^{-1} G_{n} g=G_{n} \text { for } g \in O(2) .
$$

For a subset $A$ of $O(2)$, let $\langle A\rangle$ denote the group generated by $A$. Let $1<n<$ $m<k$ be integers such that $k$ is the least common multiple of $n$ and $m$. Then it holds that

$$
\left\langle G_{n} \cup G_{m}\right\rangle=G_{k} .
$$

Indeed, using $r(\theta)$ before (1.5), we get

$$
\left\langle G_{n} \cup G_{m}\right\rangle=\{r(2 i \pi / n+2 j \pi / m): i, j \in \mathbb{Z}\} .
$$

Since $k$ is the least common multiple, there exist $i, j \in \mathbb{Z}$ such that $i / n+j / m=$ $1 / k$. Hence (6.18) holds.

Let $G_{n_{i}}$ and $u_{i}$ be as in Example 3.3. We shall show that $u_{i}$ is not equivalent to $u_{j}$ if $i \neq j$. Let $i<j$. If $n_{j}$ is a multiple of $n_{i}, G_{n_{i}}$ is a subgroup of $G_{n_{j}}$ and $G_{n_{i}}(x) \varsubsetneqq G_{n_{j}}(x)$ for $x \in \Gamma\left(G_{n_{j}}\right)$. Therefore $R\left(u_{i}\right)<R\left(u_{j}\right)$.

Let $n_{j}$ be not a multiple of $n_{i}$. The least common multiple of them is equal to an $n_{k}$ with a certain $k \leq d$. Then $R\left(u_{i}\right), R\left(u_{j}\right)<R\left(u_{k}\right)$. In contradiction to our claim, we assume that $u_{i}$ is equivalent to $u_{j}$, i.e. $u_{i}(g x)=u_{j}(x)$ with some $g \in O(2)$. Then $u_{j}$ is $g^{-1} G_{n_{i}} g$ invariant because

$$
u_{j}\left(g^{-1} h g x\right)=u_{i}(h g x)=u_{i}(g x)=u_{j}(x) \quad \text { for } h \in G_{n_{i}} .
$$

Thus $u_{j}$ is invariant under both $G_{n_{j}}$ and $g^{-1} G_{n_{i}} g$. From (6.17) and (6.18), it follows that

$$
\left\langle G_{n_{j}} \cup g^{-1} G_{n_{i}} g\right\rangle=\left\langle G_{n_{j}} \cup G_{n_{i}}\right\rangle=G_{n_{k}} .
$$

Hence $u_{j}$ is $G_{n_{k}}$ invariant. However this contradicts $R\left(u_{j}\right)<R\left(u_{k}\right)$. Therefore $u_{i}$ is not equivalent to $u_{j}$.

Proof of Example 3.4. Let $G_{n}$ be as in (1.5). We define a subgroup $H_{n}$ of $O(3)$ by

$$
H_{n}:=\left\{\left(\begin{array}{cc}
g & 0 \\
0 & 1
\end{array}\right): g \in G_{n}\right\}
$$


Then $H_{n}$ is a subgroup of $H_{2 n}$ and $H_{n}(x) \varsubsetneqq H_{2 n}(x)$ for $x \in \Gamma\left(H_{2 n}\right)$. Let $u_{n}$ be an $H_{n}$ invariant least energy solution. Fix $n$ arbitrary. Assume either (A) with $a$ sufficiently close to $\alpha$ or (B) with $\lambda>0$ large enough. Then

$$
R\left(u_{1}\right)<R\left(u_{2}\right)<R\left(u_{4}\right)<\ldots<R\left(u_{2^{n}}\right) .
$$

Therefore the assertion of Example 3.4 holds.

\section{REFERENCES}

[1] N. Ackermann, M. Clapp And F. Pacella, Self-focusing multibump standing waves in expanding waveguides, Milan J. Math. 79 (2011), 221-232.

[2] D. Arcoya, Positive solutions for semilinear Dirichlet problems in an annulus, J. Differential Equations 94 (1991), 217-227.

[3] M.A. Armstrong, Groups and symmetry, Springer, New York, 1988.

[4] M. Badiale And E. SERRA, Multiplicity results for the supercritical Hénon equation, Adv. Nonlinear Stud. 4 (2004), 453-467.

[5] T. Bartsch, M. Clapp, M. Grossi and F. Pacella, Asymptotically radial solutions in expanding annular domains, Math. Ann. 352 (2012), 485-515.

[6] T. BARtch, M. Schneider And T. Weth, Multiple solutions of a critical polyharmonic equation, J. Reine Angew. Math. 571 (2004), 131-143.

[7] V. Barutello, S. Secchi and E. Serra, A note on the radial solutions for the supercritical Hénon equation, J. Math. Anal. Appl. 341 (2008), 720-728.

[8] A. Borel, Le plan projectif des octaves et les sphères comme espaces homogènes, C.R. Acad. Sci. Paris 230 (1950), 1378-1380.

[9] J. Byeon, Existence of many nonequivalent nonradial positive solutions of semilinear elliptic equations on three-dimensional annuli, J. Differential Equations 136 (1997), 136165.

[10] J. ByeOn, S. ChO AND J. PARK, On the location of a peak point of a least energy solution for Hénon equation, Discrete Contin. Dyn. Syst. 30 (2011), 1055-1081.

[11] J. BYEON AND K. TANAKA, Multi-bump positive solutions for a nonlinear elliptic problem in expanding tubular domains, Calc. Var. PartialDifferential Equations 50 (2014). 365397.

[12] J. Byeon And Z.-Q. WANG, On the Hénon equation: asymptotic profile of ground states, I, Ann. Inst. H. Poincaré Anal. Non Linéaire 23 (2006), 803-828.

[13] - On the Hénon equation: Asymptotic profile of ground states, II. J. Differential Equations 216 (2005), 78-108.

[14] M. Calanchi, S. Secchi and E. Terraneo, Multiple solutions for a Hénon-like equation on the annulus, J. Differential Equations 245 (2008), 1507-1525.

[15] D. CaO And S. Peng, The asymptotic behaviour of the ground state solutions for Hénon equation, J. Math. Anal. Appl. 278 (2003), 1-17.

[16] D. Castorina and F. Pacella, Symmetry of positive solutions of an almost-critical problem in an annulus, Calc. Var. Partial Differential Equations 23 (2005), 125-138.

[17] F. Catrina And Z.-Q. Wang, Nonlinear elliptic equations on expanding symmetric domains, J. Differential Equations 156 (1999), 153-181.

[18] J.-L. Chern AND C.-S. Lin, The symmetry of least-energy solutions for semilinear elliptic equations, J. Differential Equations 187 (2003), 240-268.

[19] C.V. Coffman, A nonlinear boundary value problem with many positive solutions, J. Differential Equations 54 (1984), 429-437. 
[20] H.S.M. Coxeter, Regular Polytopes, third edition, Dover, New York, 1973.

[21] E.N. DANCER, On the number of positive solutions of some weakly nonlinear equations on annular regions, Math. Z. 206 (1991), 551-562.

[22] _ Global breaking of symmetry of positive solutions on two-dimensional annuli, Differential Integral Equations 5 (1992), 903-913.

[23] Some singularly perturbed problems on annuli and a counterexample to a problem of Gidas, Ni and Nirenberg, Bull. London Math. Soc. 29 (1997), 322-326.

[24] E.N. Dancer And S. YAn, Multibump solutions for an elliptic problem in expanding domains, Comm. Partial Differential Equations 27 (2002), 23-55.

[25] P. Esposito, A. Pistoia And J. Wei, Concentrating solutions for the Hénon equation in $\mathbb{R}^{2}$, J. Anal. Math. 100 (2006), 249-280.

[26] V.V. Gorbatsevich, A.L. Onishchik and E.B. Vinberg, Foundations of Lie Theory and Lie Transformation Groups, Springer, Berlin, 1997.

[27] N. Hirano, Existence of positive solutions for the Hénon equation involving critical Sobolev terms, J. Differential Equations 247 (2009), 1311-1333.

[28] R. KAJikiYA, Orthogonal group invariant solutions of the Emden-Fowler equation, Nonlinear Anal. 44 (2001), 845-896.

[29] _ Least energy solutions of the generalized Hénon equation in reflectionally symmetric or point symmetric domains, J. Differential Equations 253 (2012), 1621-1646.

[30] L Least energy solutions of the Emden-Fowler equation in hollow thin symmetric domains, J. Math. Anal. Appl. 406 (2013), 277-286.

[31] __ Least energy solutions without group invariance for the generalized Hénon equation in symmetric domains, Submitted for publication.

[32] _ Multiple positive solutions of the Emden-Fowler equation in hollow thin symmetric domains, Calc. Var. Partial Differential Equations 52 (2015), 681-704.

[33] A. KRistály and W. Marzantowicz, Multiplicity of symmetrically distinct sequences of solutions for a quasilinear problem in $\mathbb{R}^{N}$, NoDEA Nonlinear Differential Equations Appl. 15 (2008), 209-226.

[34] Y.Y. LI, Existence of many positive solutions of semilinear elliptic equations in annulus, J. Differential Equations 83 (1990), 348-367.

[35] S.-S. Lin, Positive radial solutions and non-radial bifurcation for semilinear elliptic equations in annular domains, J. Differential Equations 86 (1990), 367-391.

[36] _ Existence of positive nonradial solutions for nonlinear elliptic equations in annular domains, Trans. Amer. Math. Soc. 332 (1992), 775-791.

[37] _ Existence of many positive nonradial solutions for nonlinear elliptic equations on an annulus, J. Differential Equations 103 (1993), 338-349.

[38] _ Asymptotic behavior of positive solutions to semilinear elliptic equations on expanding annuli, J. Differential Equations 120 (1995), 255-288.

[39] N. Mizoguchi, Generation of infinitely many solutions of semilinear elliptic equation in two-dimensional annulus, Comm. Partial Differential Equations 21 (1996), 221-227.

[40] N. Mizoguchi And T. Suzuki, Semilinear elliptic equations on annuli in three and higher dimensions, Houston J. Math. 22 (1996), 199-215.

[41] D. Montgomery and H. Samelson, Transformation groups of spheres, Ann. of Math. 44 (1943), 454-470.

[42] D. Montgomery and L. Zippin, Topological Transformation Groups, Robert E. Krieger Publishing, New York, 1974. 
[43] M. Musso, F. PACARD AND J. WEI, Finite-energy sign-changing solutions with dihedral symmetry for the stationary nonlinear Schrödinger equation, J. Eur. Math. Soc. 14 (2012), 1923-1953.

[44] A.L. Onishchik, Topology of Transitive Transformation Groups, Johann Ambrosius Barth, Leipzig, 1994.

[45] F. PACARD, Radial and nonradial solutions of $-\Delta u=\lambda f(u)$, on an annulus of $\mathbb{R}^{n}, n \geq 3$, J. Differential Equations 101 (1993), 103-138.

[46] A. Pistoia And E. Serra, Multi-peak solutions for the Hénon equation with slightly subcritical growth, Math. Z. 256 (2007), 75-97.

[47] L.S. Pontruagin, Topological Groups, third edition. translated from the Russian by A. Brown, Gordon and Breach Science Publishers, New York, 1986.

[48] E. Serra, Non radial positive solutions for the Hénon equation with critical growth, Calc. Var. Partial Differential Equations 23 (2005), 301-326.

[49] D. Smets, M. Willem AND J. Su, Non-radial ground states for the Hénon equation, Commun. Contemp. Math. 4 (2002), 467-480.

[50] T. Suzuki, Positive solutions for semilinear elliptic equations on expanding annuli: mountain pass approach, Funkc. Ekvac. 39 (1996), 143-164.

[51] Z.-Q. WANG AND M. WILlEM, Existence of many positive solutions of semilinear elliptic equations on an annulus, Proc. Amer. Math. Soc. 127 (1999), 1711-1714.

Manuscript received April 25, 2013

RYuJi KAJIKIYA

Department of Mathematics

Faculty of Science and Engineering

Saga University

Saga, 840-8502, JAPAN

E-mail address: kajikiya@ms.saga-u.ac.jp 